\title{
From Drop-Shape Analysis to Stress-Fitting Elastometry
}

\section{Journal Article}

\section{Author(s):}

Nagel, Mathias; Tervoort, Theo A. (D); Vermant, Jan

Publication date:

2017-09

Permanent link:

https://doi.org/10.3929/ethz-b-000189251

\section{Rights / license:}

Creative Commons Attribution-NonCommercial-NoDerivatives 4.0 International

\section{Originally published in:}

Advances in Colloid and Interface Science 247, https://doi.org/10.1016/j.cis.2017.07.008 


\title{
From Drop-Shape Analysis to Stress-Fitting Elastometry
}

\author{
Mathias Nagel $^{\mathrm{a}}$, Theo A. Tervoort ${ }^{\mathrm{a}}$, Jan Vermant ${ }^{\mathrm{a}, *}$ \\ ${ }^{a}$ Department of Materials, Vladimir-Prelog-Weg 5, ETH Zurich, 8093 Zurich, Switzerland
}

\begin{abstract}
Drop-shape analysis using pendant or sessile drops is a well-established experimental technique for measuring the interfacial or surface tension, and changes thereof. The method relies on deforming a drop by either gravity or buoyancy and fitting the Young-Laplace equation to the drop shape. Alternatively one can prescribe the shape and measure the pressure inside the drop or bubble using pressure tensiometry. However, when an interface with a complex microstructure is present, extra and anisotropic interfacial stresses may develop due to lateral interactions between the surface-active moieties, leading to deviations of the drop shape or even a wrinkling of the interface. To extract surface-material properties of these complex interfaces using drop-shape analysis or pressure tensiometry, the Young-Laplace law needs to be generalized in order to account for the extra and anisotropic stresses at the interface. In the present work we review the different approaches that have been proposed to date to extract the surface tension as the thermodynamic state variable, as well as other rheological material properties such as the compression and the shear modulus. To evaluate the intrinsic performance of the methods, computer generated drops are subjected to step-area changes and then subjected to analysis using the different methods. Shape-fitting methods, now combined with an adequate constitutive method, do however perform rather poorly in determining the elastic stresses, especially at small area strains. An additional measurement of the pressure or capillary-pressure tensiometry is required to improve the sensitivity. However, pressure-based methods still require the knowledge of the undeformed reference state, which may be difficult to achieve in practice. Moreover, it is not straightforward to judge from what point onwards one needs to go beyond the Young-Laplace equation. To overcome these limitations, a method based on stress fitting, which uses a local force balance method, is introduced here. One aspect of this new method is the use of the Chebyshev transform to numerically describe the contour shape of the drop interface. For all methods we present a detailed error analysis to evaluate if, and with what precision, surface material parameters can be extracted. Depending on the desired information, different ideal experimental conditions and most suitable methods are discussed, in addition to having a criterion to investigate if extra and anisotropic stresses matter.
\end{abstract}

Keywords: Pendant Drop, Interfacial Rheology, Surface Tension, (Axisymmetric) Drop Shape Analysis - DSA, Stress-fitting elastometry - SFE

\section{Introduction}

\subsection{Pendant drop tensiometry: success and pitfalls}

The analysis of the shape of pendant or sessile drops or bubbles, has developed into an important and standard technique in the interfacial science of fluid-fluid interfaces. Such investigations are concerned with the measurement of surface tension of a liquid-air interface, interfacial tension between two liquids and with the mechanical and physicochemical behavior of adsorbed substances at such interfaces.

The interfacial properties are derived from the shape of drops or bubbles, an idea first presented by Worthington, as early as 1881 [1]. He projected the silhouette of a pendant drop on a paper screen, o sketched its contour and evaluated the curvature using a graphical method. Worthington recognized that there is a balance between the hydrostatic pressure, which changes with height and the Laplace

\footnotetext{
* Corresponding author

Email address: jan.vermant@mat.ethz.ch (Jan Vermant )

$U R L$ : www.softmat.mat.ethz.ch (Jan Vermant ) 
pressure caused by the presence of a curved interface.

The Young-Laplace equation expresses this balance:

$$
\left(\kappa_{1}+\kappa_{2}\right) \sigma_{\alpha \beta}=p-\rho g z .
$$

Herein $\kappa_{1}$ and $\kappa_{2}$ are the two principal curvatures, $\sigma_{\alpha \beta}$ the surface or interfacial tension ${ }^{1}, p$ the pressure 15 difference over the interface at the vertical position $z=0, \rho$ the density difference and $g$ the gravitational acceleration.

Due to the non-linear dependency of the curvatures on the drop radius and height, the application of the Young-Laplace equation in a wparameter fitting process is complicated. It was cast into its modern form by Rotenberg et al. in 1983 [2], who developed an algorithm that compared numerically sampled drop shapes to computationally obtained profiles. Computer assisted iterative fitting allowed to use many points on the interface and improved the precision compared to fitting of only few characteristic points to tabulated data as done in the pre-computer age $[3,4]$.

Since then, numerical fitting has been subject to constant improvements in terms of robustness, efficiency and precision. Provided that robust numerical schemes are used, Axisymmetric Drop Shape Analysis (ADSA or DSA) developed in the group of Neumann [5] is an accurate method for the determination of the surface tension of pure surface-tension interfaces by fitting the drop shape to a solution of the Laplace equation of undetermined surface tension [6]. A key aspect for the method to work is that so the drop needs to be deformed enough by gravity $[7,8]$. Without a hydrostatic pressure contribution the drop is spherical and the curvature does not vary. As a consequence only the ratio between surface tension and pressure is fixed, but not their absolute values.

A variation of the DSA method is the capillary-pressure tensiometer (CPT) introduced by Sudgen in 351922 [9] and the pulsating-bubble surfactometer (PBS) [10]. Both methods couple a shape analysis with a pressure measurement, using generally smaller capillaries to increase the pressure signal. The shape analysis simplifies considerably when the capillary pressure is provided, as only the curvature has to be determined. So for the CPT method having a near spherical or hemispherical shape is an advantage as the curvature is then easily determined. Recently Peters et al. [11] presented a new approach based on a 40 force balance, which is expected to be more robust, because it requires only the tangent angle (derivative) of the interface and not the curvature (double derivative). Applying a derivative to experimental data is known to amplify noise.

DSA was originally developed for the determination of the surface tension, but it has been used for interfacial tensions between immiscible liquids [12] even for very small values [13] or transient conditions $[14,15]$. DSA has also been applied to a film balance for insoluble substances $[16,17]$. However, when going to interfaces which become "complex", due to the presence of an interfacial structure with lateral interactions between the interfacial moieties, there will be a corresponding mechanical response particular to the interface. A scalar value of the surface tension will no longer suffice to describe the properties of that interface and the shape of pendant drops deviates from a Laplacian one. A striking example of this was, for example, seen in interfacial layers from the protein HFBII hydrophobin, where an increase in the error of the fit of the pendant-drop profile by means of the Laplace equation was observed as the layers were expected to undergo a transition from fluid to elastic solid films [18], with interfaces even being observed to crumple. In the case of evaporating drops with asphaltene particles at the inter5 face buckling has also been observed, where an inverted curvature indicates a build-up of compressive stresses [19]. Also these observations indicate that an analysis is required which goes beyond the mere usage of surface tension. Botto et al. [20] used simulations of particle-laden drops and showed how the microstructure-surface stress relationship influences the drop shape. In particular they showed how the isotropic and anisotropic surface stress stem from the interparticle forces and the organization at the 60 interface for the case of repulsive spheres.

In the following we present a review of the different approaches which have been developed to tackle the problem of static drops or bubbles in presence of an elastic, structured interfaces, in particular

\footnotetext{
${ }^{1}$ The subscript $\alpha \beta$ is not an index but rather clarifies that the stress $\sigma$ is due to the presence of two adjoints $\alpha$ and $\beta$.
} 
subjected to step area changes. We extend the analysis and provide robust algorithms to obtain the material parameters, with some guidelines for the best experimental conditions. The analysis is also relevant for oscillatory pendant drop experiments, when the interface is viscoelastic. The static case for elastic interfaces represents the limit of a high Deborah number. In that case, hydrodynamics and mass transfer kinetics may obviously lead to additional complexity, see for example Alvarez et al. [21] et al. and Balemans et al. [22] for recent accounts accounts on mass transfer and momentum transfer effects, respectively.

\subsection{Pendant drop elastometry}

Carvajal et al. [23] were the first to take the pendant drop problem beyond a simple interface, introducing an interfacial shell with solid-like behavior in addition to the surface tension. Due to the presence of the elastic shell, at any given point of the interface, the two principal interfacial stresses are no longer

75 necessarily equal to one other. Using the assumption of an incompressible volume preserving interfacial layer, their isochoric model interface was characterized by a single parameter, the Young's modulus. The deviations between a Young-Laplace fit and the resulting elastic shape were studied by Ferri et al. [24] theoretically and experimentally. They investigated the influence of the interfacial shear modulus on the fit error of the Young-Laplace equation. However, when solving the inverse problem they used only the

${ }_{80}$ isotropic Young-Laplace equation to obtain the surface tension. With the assumption of Poisson ratio $\nu=0$ (when compression and shear modulus are equal, see appendix A. for definitions) the authors obtained the surface shear modulus $G$. This seems a very strong assumption, as the shear modulus $G$ captures the reaction of the interface to a change in shape (deviatoric stress), and is at the heart of the anisotropic stress distribution at the interface. Close to the neck of the capillary the interfacial fluid

85 elements will always deform and coupling $G$ artificially to $K$ by supposing $\nu=0$ may not give physically realistic results for all interfaces.

Work by Knoche et al. [25] extended Carvajals analysis of elastic interfaces to two parameter models, using a Young's modulus and the Poisson ratio. Their work investigates hydrophobin (HFBII) and

90 polymerized octadecyltrichlorosilane (OTS) layers under compression and also relates the number of wrinkles of the membrane to the bending modulus. Knoche et al. observed large uncertainties on the Poisson ratio $\nu$, for example for HFBII drops. Their 2-parameter model is only valid in the small-strain limit.

Vaccari et al. studied elastic biofilms with a pendant drop and also analyzed the shear modulus with 95 micro-rheology particle tracking. Their curve fitting also led to a wide spread of data for the Poisson ratio. Danov et al. [26] showed that in these cases the analysis of material properties relies on the knowledge on the unstrained reference state. They developed a method which they called Capillary Meniscus Dynamometry (CMD) to determine the anisotropic stress state at the interface, however no attempt was made to extract material properties from the stress state.

The work of Carvajal [23] also included a discussion on the most favorable experimental conditions. The precision of the pendant drop method faces stronger inherent limitations than other established techniques like the Langmuir trough. For example, the actual straining of the interface (albeit a complex deformation, as first shown by Petkov et al. [27]) is better prescribed in the Langmuir trough. In a pendant drop this will only be obtained as a result of the analysis used to extract the material parameters. This increased uncertainty generally requires larger strains and in return might actually be outside the linear regime, which is assumed in most of the approaches used so far. Moreover, experimental determinations of the surface deformation via optical methods also require substantial area deformations.

When the surface stress becomes very large it will be capped at the surface tension of the bare interface and wrinkles form to relax the compressive stress. Knoche et al. [25] were able to accurately predict where wrinkles would form based on elastic theory. As we are however, interested in a precise determination of the linear response, the issues of wrinkling and bending of the interface will not be pursued here. The current state of the art can be found in ref. [25].

\subsection{Terminology and Definitions}

A pendant-drop apparatus has a hardware part for the drop control and optical-image acquisition and a software part for the image treatment. The first subsystem concerns the optical equipment and dispensing systems. Since this remains almost unchanged whether isotropic or elastic interfaces are used, 
there is a large body of literature and reviews available [6, 8] and we shall focus on the data processing. To line up the terminology we specify that the drop-shape analysis is composed of shape sampling, which is the process of obtaining a numerically sampled shape from a drop image, and parameter fitting, which is the process of obtaining the material parameters from a sampled shape.

We differentiate between different types of drop analysis and propose a unified vocabulary in order classify the increasing number of names that appear in the literature. Pendant-drop tensiometry measures the interfacial tension, where isotropic tensiometry, like DSA, retrieves the surface tension $\sigma_{\alpha \beta}(\Gamma)$ with $\Gamma$ the surface concentration of a surface active substance. Further, anisotropic tensiometry, like the CMD method, retrieves the anisotropic surface stresses. Pendant-drop elastometry is proposed here as the name for a shape-analysis method that aims to retrieve elastic material parameters. In a more generalized fashion one should use the term pendant-drop rheometry if viscoelastic material functions are investigated.

Furthermore, the methodologies can be divided into pressure-assisted and pure shape-fitting methods, depending on whether the pressure is obtained by a pressure sensor or calculated from the curvatures in a shape fitting routine. For example, CPT is pressure-assisted tensiometry which assumes isotropic deformations. Based on the terminology we classify the approach developed by Carvajal et al. [23] and Knoche et al. [25] as Drop-Shape Fitting Elastometry. In this work we will review and extend their approach to include the independently measured pressure in the capillary and name this consequently Pressure-Assisted Elastometry.

Finally, the different methods differ in the numerical scheme to solve the inverse problem of the parameter fitting. We categorize into three families: 1. direct methods that integrate the sampled coordinates directly, 2. iterative-decoupled methods that iteratively fit the interface shape and separately optimize the parameters, and 3. fully coupled inverse methods. In order to validate the different algorithms, we make use of numerically generated drop shapes. If the fitting is made directly from such "synthetic" shapes, we write synthetic-numeric and if the fitting is made by shape fitting to a grey-value image created from a synthetic shape we write synthetic-image. Synthetic-image fitting allows to evaluate the performance of the shape-sampling routines and the parameter-fitting routines. Synthetic images are created with a resolution of 1000 pixel at the widest drop diameter, which corresponds roughly to standard imaging equipment.

Bold upper-case letters (A) are used to indicate matrices and bold lower-case letters (a) indicate vectors or one dimensional arrays. Bold Greek letters are vectors or arrays except for $\boldsymbol{\sigma}$ and $\boldsymbol{\sigma}_{\boldsymbol{e}}$, which are second order tensors. Plain characters are scalars of scalar valued functions, where a subscript $i, n$ which denotes the extra stress and Roman number subscripts $\left(\sigma_{I I}\right)$, which distinguishes between different instances of the same variable, for example belonging to two different deformed states. Superscript indices $s, r, d$ and $i s o$ are used only for the stress $\sigma$, stretch $\lambda$ and curvature $\kappa$ and indicate the direction of the respective quantity or whether the quantity is deviatoric or isotropic. A star superscript (as in radius $r^{\star}$ ) denotes a variable that belongs to an isotropic reference state.

The surface stress tensor $\boldsymbol{\sigma}=\sigma_{\alpha \beta}(\Gamma) \mathbf{I}+\boldsymbol{\sigma}_{\boldsymbol{e}}$ at a fluid-fluid interface is composed of an isotropic surface tension contribution $\sigma_{\alpha \beta} \mathbf{I}$ and extra and deviatoric stresses $\boldsymbol{\sigma}_{\boldsymbol{e}}$. Consequently, the total isotropic stress is characterized by a concentration-dependent state variable, namely the surface tension $\sigma_{\alpha \beta}(\Gamma)$, and variables which describe the mechanical or rheological contribution coming from a (visco)elastic interface, $\operatorname{tr}\left(\boldsymbol{\sigma}_{\boldsymbol{e}}\right) / 2$, where $\operatorname{tr}()$ denotes the trace (first invariant) of a tensor. The state variable surface tension $\sigma_{\alpha \beta}(\Gamma)=\sigma_{\alpha \beta, 0}-\Pi(\Gamma)$ is composed of the equilibrium surface tension $\sigma_{\alpha \beta, 0}$ of the pristine, clean interface and a surface pressure $\Pi(\Gamma)$ that depends on species concentration $\Gamma$ at the interface. Apart from the isotropic extra stresses there can also be deviatoric extra stresses $\boldsymbol{\sigma}_{\boldsymbol{e}}^{\boldsymbol{d}}=\boldsymbol{\sigma}_{\boldsymbol{e}}-\frac{1}{2} \operatorname{tr}\left(\boldsymbol{\sigma}_{\boldsymbol{e}}\right) \mathbf{I}$, that builds up when the principal strains are not equal. Under static conditions, a simple or fluid-like interface can be adequately described by the Young-Laplace equation, whereas an interface with elastic deviatoric stresses needs a more elaborate mathematical description. In practise it is not always possible to separate the isotropic contribution into a component related to the thermodynamic state variable and one related to the mechanical material properties or functions, as this requires experiments where the 
another).

\subsection{Structure of this paper}

In section 2 the definition of the geometrical representation of the interface is given and its sampling in terms of Chebyshev polynomials is discussed. Section 2.2 presents the governing equations of an interface which can support both isotropic and deviatoric stresses is presented. How to compute drop shapes and how to retrieve the material parameter from given shapes is explained in sections 3.1 and 3.3 for isotropic interfaces and in sections 3.5 and 3.7 for anisotropic elastic interfaces. Finally, in section 4 we compare the performance of the different methods for isotropic and elastic interfaces. We then summarize

\section{Problem definition}

Since the representation of the interface is at the core of the drop-shape analysis, the used coordinate system is introduced first. A cylindrical coordinate system is used with a radial axis $r$, azimuthal angle $\varphi$ and a vertical axis $z$ that is oriented in the opposite direction of the gravitational force. In the following, we shall use the term "drop" as a generic descriptor for pendant and sessile drops as well as for rising bubbles. What is said about interfacial tension can be used for surface tension as well. The experimentally acquired drop image is typically a contour projection in a $r-z$ plane. The use of the arc-length $s$ with $s=0$ at the apex of the drop as independent variable is almost the exclusive choice in the literature. The contour $r(s)$ and $z(s)$ are functions of $s$, where $d s=\sqrt{d r^{2}+d z^{2}}$. Using the arc-length $s$ is the natural strategy to avoid problems such as $r(z)$ or $z(r)$ being singular or multi valued. A different approach has been used by Danov et al. [26], who choose to describe the interface by a function $r^{2}(z)$, which removes the singularity at the apex but requires the use of $r^{2}$ for subsequent calculations, where $r$ seems more natural.
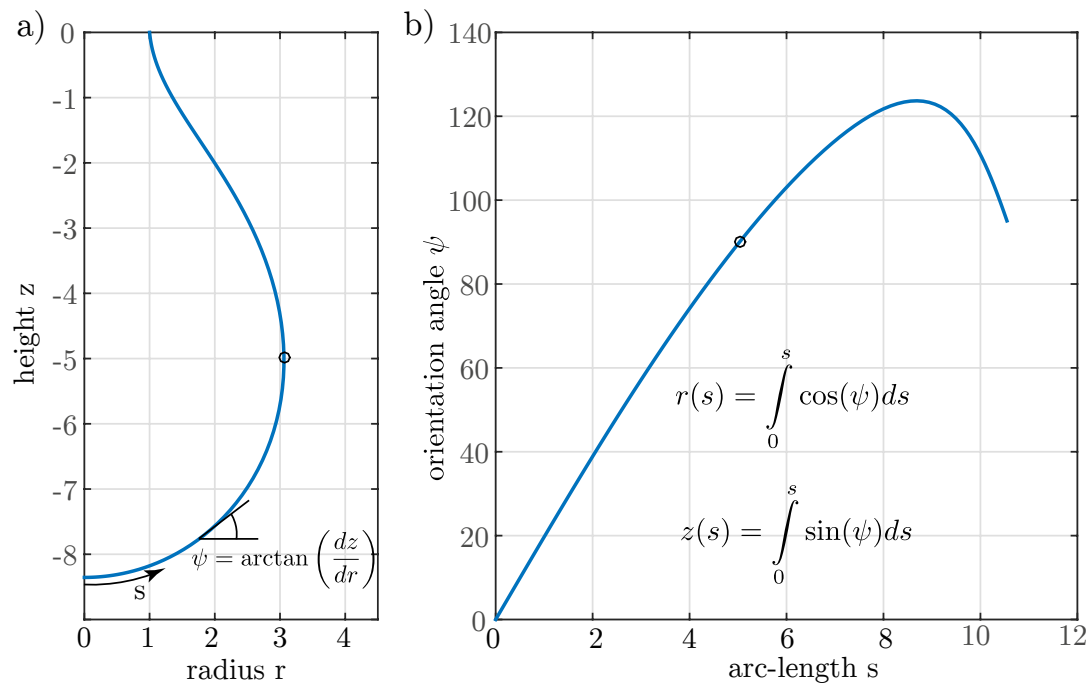

Figure 1: Representation of the geometry in coordinates $r, z$ (a) and $s, \psi(\mathrm{b})$. The location where the orientation angle is $90^{\circ}$ is marked by a circle in both figures.

Instead of coordinates $(r, z)$ the interface can independently be described by $(\psi, s)$, where $(\psi)$ is the tangent angle. The coordinate systems are related by:

$$
\frac{d r}{d s}=\cos (\psi), \quad \frac{d z}{d s}=\sin (\psi)
$$

Although $\psi$ is related in a non-linear way to $r$ and $z$ it simplifies the expressions for the curvature:

$$
\kappa^{s}=-\frac{\frac{d^{2} r}{d s^{2}}}{\sqrt{1-(d r / d s)^{2}}}=\frac{d \psi}{d s}, \quad \kappa^{r}=\frac{1}{r} \frac{d z}{d s}=\frac{\sin (\psi)}{r}
$$


An example Young-Laplace shape in both coordinate systems is plotted in Figure 1. In the case of continuous variables we shall denote the radius at apex location $r_{a}$, radius of the interface near the capillary tip $r_{t}$ and the radius of the capillary $r_{c}$. In the discrete case the drop radius is given by $N$ number of points: $r_{i}=r_{1} \ldots r_{N}$. The same holds for the other coordinates $s, z$ and $\psi$. We chose the origin of the coordinate systems such that $s_{a}=s_{1}=0, r_{a}=r_{1}=0$ and $z_{t}=z_{N}=0$. From symmetry it follows that $\psi_{a}=\psi_{1}=0$.

\subsection{Sampling the interface with a Chebyshev transform}

When sampling the image of a drop into a numeric array during the shape-sampling step, the objective is to achieve good precision with the least number of points. As we will see later on in section 3.7 it is also important, for example to calculate $\psi$, to have a numerical representation of the interface that is free of spurious oscillations, in particular close to the point of attachment of the interface to the capillary. Oscillations appear when noise is amplified in numerical differentiation. It is actually more pronounced when the sampling is denser.

The interface location is obtained by scanning the 2D image array along vertical and horizontal lines. Grey-value interpolation between pixels the sub-pixel is the used to determine the interface location by pairs of $R_{i}$ and $Z_{i}$. From these pairs $\Delta S_{i}$ is computed as the distance between consecutive pairs : $\Delta S=\sqrt{\left(R_{i}-R_{i-1}\right)^{2}+\left(Z_{i}-Z_{i-1}\right)^{2}}$. Finally $\Delta S_{i}$ is summed up for each step $i$, which adds to each pair $R_{i}, Z_{i}$ their unique arc-length $S_{i}$.

To obtain a robust description of the interface an orthogonal basis of Chebyshev polynomials is proposed, as these functions are well suited to approximate a smooth function on a finite size domain [28]. Additionally, Chebyshev polynomials are a natural basis for the spectral method that solves differential equations with an exponential convergence. In order to transform a shape $f(s)$ into Chebyshev modes $a_{k}$ :

$$
a_{k \geq 0}=\int_{-1}^{1} \frac{p_{k}}{\pi} \frac{f(t) T_{k}(t)}{\sqrt{1-t^{2}}} d t, \quad p=1, p_{k>0}=2, t=2 \frac{s}{s_{t}}-1 .
$$

Where $t$ is a normalization of $s=0 \ldots s_{N}$ on the basis $-1 \ldots 1$ and $T_{k}(t)$ is the Chebyshev polynomial of the first kind of order $k$. The Chebyshev filtered function $\hat{f}(t)$ is then retrieved by $\hat{f}(t)=\sum_{k=0}^{n} a_{k} T_{k}(t)$.
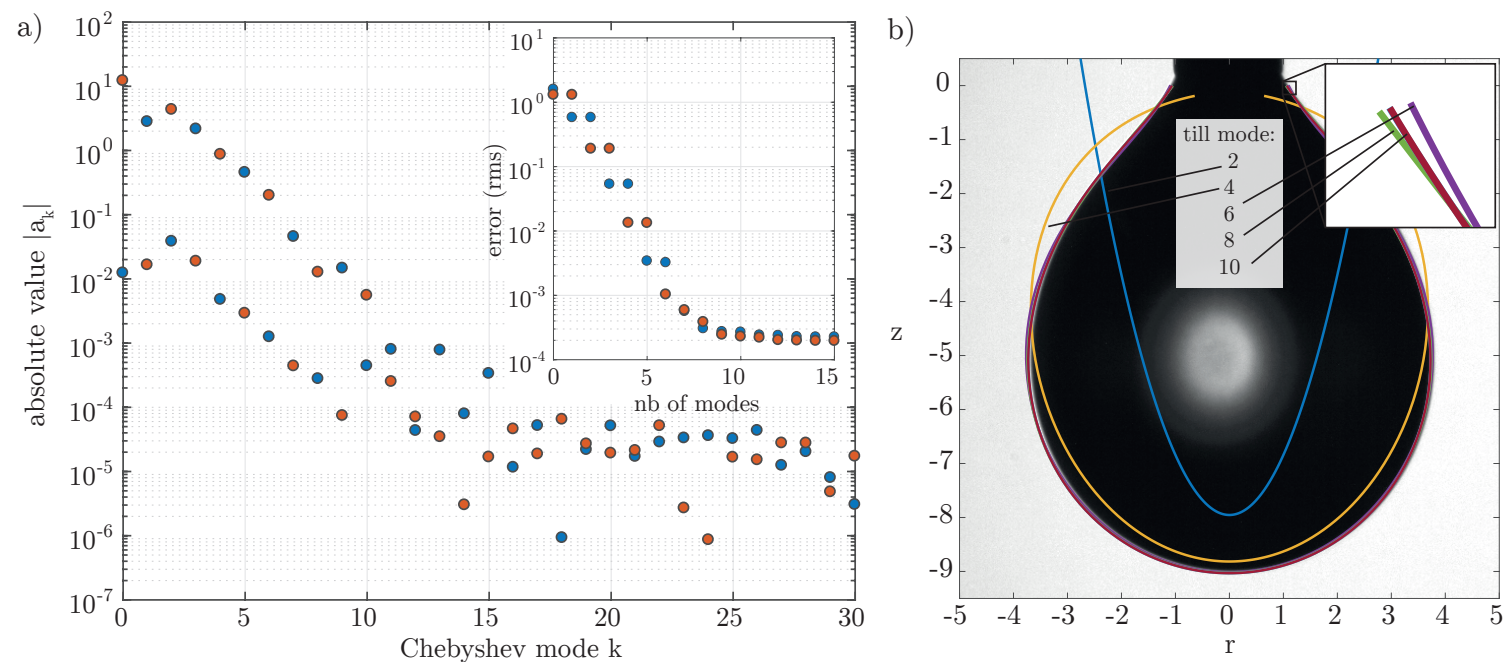

Figure 2: Chebyshev fit of a drop shape from a water drop in air taken from the data [7]. The absolute values of the fit are shown in Figure (a). The difference between the acquired shape and the shape consisting of increasing modes is shown in the inset. Figure (b) shows the drop shape and the modal shapes for increasing order.

Projecting the shape functions $r(s)$ and $z(s)$ on Chebyshev polynomials, allows to filter spurious modes by truncation at the highest order that leads to a decreased residual. Further increasing the number of modes is not useful, as they can be thought of as representing noise and in applying a derivative on the data this noise is greatly amplified. The Chebyshev transform as a filtering technique is typically more precise than a smoothing scheme, which will degrade the shape quality and will lead to 
a loss of information. Furthermore, due to its integral character, the Chebyshev filtered $\hat{R}_{i}, \hat{Z}_{i}$ contains information of all pixels $R_{i}, Z_{i}$, whereas simple sampling of a given number of locations discards information. It therefore compresses the information. We denote the sampled geometry with capital letters and regardless whether filtering has been applied or not the hat will be omitted. In subsequent sections on fitting parameter the capital letters (e.g. $R_{i}$ ) will indicate sampled positions that constrain the computed positions (e.g. $r_{i}$ ). Although direct methods to compute the volume or surface stresses, as in section 3.3, 3.4 and 3.7, could also operate on the sampled coordinates we will use small case letters for uniformity and since no ambiguity between sampled and computed values is present.

An example is shown in Figure 2, where a drop shape image taken from Berry et al. [7] has been sampled and transformed in Chebyshev modes $a_{k}$. The absolute value of $a_{k}$ decreases exponentially, and due to the symmetry in $Z_{i}$ and antisymmetry in $R_{i}$ only every odd or even mode is non-zero. Because of imperfect alignment every second mode is only near but not exactly zero. After about 15 modes only noise is fitted. As can be seen in the inset, when comparing sampled and filtered point only the first 10 modes contribute considerably to the shape.

\subsection{Governing equations}

The analysis is pursued using non-dimensional parameters. Hereafter we will write dimensional quantities with a tilde and non-dimensional without. The natural length scale in the problem is the capillary radius $\tilde{r}_{c}$ and the subsequent natural pressure scale is the hydrostatic pressure $\tilde{\rho} \tilde{g} \tilde{r}_{c}$. The nondimensional parameters then become $r=\tilde{r} / \tilde{r}_{c}, z=\tilde{z} / \tilde{r}_{c}$ and $\kappa=\tilde{\kappa} \tilde{r}_{c}$, allowing to rewrite the dimensional Young-Laplace equation eq.(1) in non-dimensional terms as:

$$
\left(\kappa^{s}+\kappa^{r}\right) \sigma_{\alpha \beta}=p-z, \quad \text { with } \quad \sigma_{\alpha \beta}=\frac{\tilde{\sigma}_{\alpha \beta}}{\tilde{\rho} \tilde{g} \tilde{r}_{c}^{2}} \quad \text { and } \quad p=\frac{\tilde{p}}{\tilde{\rho} \tilde{g} \tilde{r}_{c}}
$$

Herein the non-dimensional surface tension $\sigma_{\alpha \beta}$ is the ratio of a hydrostatic contribution to the surface tension contribution $\tilde{\sigma}_{\alpha \beta}$, where $\tilde{\rho}=\tilde{\rho}_{\alpha}-\tilde{\rho}_{\beta}$ is the density difference between inner and outer liquid and the $\tilde{g}$ the gravitational acceleration. It is the inverse of the Bond number $(B o)$. Since we will later on consider a spatially varying surface stress, we will not non-dimensionalize with the surface tension, but rather choose to non-dimensionalize with the hydrostatic pressure, $\tilde{\rho} \tilde{g} \tilde{r}_{c}$.

When deviatoric stresses are present, the meridional and hoop stresses are no longer equal and the Young-Laplace law needs to be generalized to:

$$
\left(\kappa^{s} \sigma^{s}+\kappa^{r} \sigma^{r}\right)=p-z, \quad \text { with } \quad \sigma^{s}=\frac{\tilde{\sigma}^{s}}{\tilde{\rho} \tilde{g} \tilde{r}_{c}^{2}} \text { and } \quad \sigma^{r}=\frac{\tilde{\sigma}^{r}}{\tilde{\rho} \tilde{g} \tilde{r}_{c}^{2}}
$$

The scalar surface tension $\sigma_{\alpha \beta}$ is now replaced by two spatially varying surface stresses $\sigma^{s}(s)$ and $\sigma^{r}(s)$, which contain the surface tension as well as the extra and deviatoric stress components. Since two stresses need to be determined at each location a second equation is needed, which is the stress balance in the meridional direction [23]:

$$
r \frac{d \sigma^{s}}{d s}=\cos (\psi)\left(\sigma^{r}-\sigma^{s}\right)
$$

\subsection{Surface tension and surface stress : Elastic interfaces}

Simple interfaces are described by a surface tension that is independent of the direction when the surface concentration of the active species is constant. For complex interfaces this is no longer the case. Considering the interface as a very sharp layer, similar to excess thermodynamic properties, excess interfacial stresses can be defined $[29,30]$ which are a combination of an isotropic surface tension $\tilde{\sigma}_{\alpha \beta}$ and an extra stress contribution that consists of an isotropic $\left(\sigma^{i s o}=\operatorname{tr}\left(\boldsymbol{\sigma}_{\boldsymbol{e}}\right) / 2\right)$ and a deviatoric part:

$$
\left(\begin{array}{cc}
\tilde{\sigma}^{s} & 0 \\
0 & \tilde{\sigma}^{r}
\end{array}\right)=\left(\tilde{\sigma}_{\alpha \beta, 0}-\tilde{\Pi}(\tilde{\Gamma})+\tilde{\sigma}_{e}^{i s o}\right) \mathbf{I}+\left(\begin{array}{cc}
\tilde{\sigma}_{e}^{s}-\tilde{\sigma}_{e}^{i s o} & 0 \\
0 & \tilde{\sigma}_{e}^{r}-\tilde{\sigma}_{e}^{i s o}
\end{array}\right)
$$

Projecting the stresses onto a coordinate system in $r-s$, expresses the stress state in principal stresses with no shear-stress component. The elastic-extra stress $\boldsymbol{\sigma}_{\boldsymbol{e}}$ for elastic interfaces depends on the local deformation. 


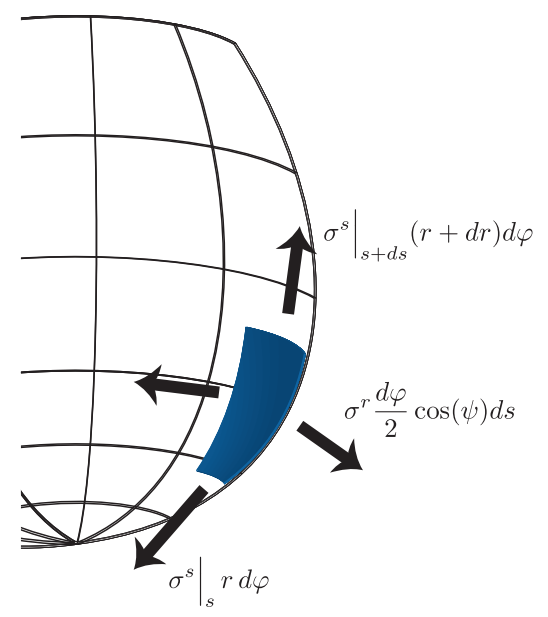

Figure 3: Illustration of the different stress contribution on an infinitesimal element.

To relate local deformations to stresses Knoche et al. [25] adopted a 2D-neo Hookean model. In the limit of small deformations the interface can be described by the dilatational modulus $K$ and a shear modulus $G$ or likewise by a $2 D$ Young's modulus $E$ and Poisson ratio $\nu$. The identities are given in Appendix A. Here we will prefer $K$ and $G$ as the stress strain relations are widely used in interfacial rheology. When transforming for the strain measure from $(E, \nu)$ into $(K, G)$ moduli the area is expressed as $\lambda^{s}+\lambda^{r}-1$, which works only for small stretches $\lambda \sim 1$. However the area is given by $\lambda^{s} \lambda^{r}$. As $K$ works against area changes and $G$ against shape changes of a surface element we can express the infinitesimal strain for $K$ as $\lambda^{s} \lambda^{r}-1$ and the shape change for $G$ as $\lambda^{s} / \lambda^{r}-1$.

Knoche et al. [25] used a model, which is only valid for infinitesimal strains which is exactly where the inverse problem of shape fitting is poorly defined. To account for the larger deformation encountered in experiments and typically required, the use of finite strain measures, like the Hencky strain, is known to correctly incorporate modest strain non-linearities [31], even though at large deformations it might result in erroneous behavior [32]. A specific advantage of the Hencky strain is that it is symmetric with respect to extension and recompression.

Using the Hencky strain measure the isotropic dilatational stress is given by:

$$
\sigma^{i s o}=-\tilde{\pi}(\Gamma)+\frac{1}{2} \operatorname{tr}\left(\tilde{\boldsymbol{\sigma}}_{e}\right)=\tilde{K} d \ln (a)=\tilde{K} \ln \left(\lambda^{s} \lambda^{r}\right) .
$$

Here the deformation is characterized by $\lambda^{s}=d \tilde{s} / d \tilde{s}^{\star}$ and $\lambda^{r}=\tilde{r} / \tilde{r}^{\star}$, where values with a star denote the isotropic reference state. The product of $\lambda^{s} \lambda^{r}$ is then equal to the relative area deformation $a / a^{*}$. The deviatoric contribution $\left(\tilde{\boldsymbol{\sigma}}_{\boldsymbol{e}}-1 / 2 \operatorname{tr}\left(\tilde{\boldsymbol{\sigma}}_{\boldsymbol{e}}\right) \mathbf{I}\right)$ stems from a change in aspect-ratio of a surface element, which is related to the deformation as:

$$
\tilde{\sigma}_{e}^{s}-\frac{1}{2} \operatorname{tr}\left(\tilde{\boldsymbol{\sigma}}_{\boldsymbol{e}}\right)=\tilde{G} \ln \left(\frac{\lambda^{s}}{\lambda^{r}}\right), \quad \tilde{\sigma}_{e}^{r}-\frac{1}{2} \operatorname{tr}\left(\tilde{\boldsymbol{\sigma}}_{\boldsymbol{e}}\right)=\tilde{G} \ln \left(\frac{\lambda^{r}}{\lambda^{s}}\right) .
$$

After non-dimensionalizing the stresses and moduli with the hydrostatic pressure scale, e.g. $K=\tilde{K} / \rho g r_{c}^{2}$, we obtain a pseudo-linear Hookean model:

$$
\begin{aligned}
\sigma^{s}-\sigma_{\alpha \beta}^{\star} & =K \ln \left(\lambda^{s} \lambda^{r}\right)+G \ln \left(\frac{\lambda^{s}}{\lambda^{r}}\right), \\
\sigma^{r}-\sigma_{\alpha \beta}^{\star} & =K \ln \left(\lambda^{s} \lambda^{r}\right)+G \ln \left(\frac{\lambda^{r}}{\lambda^{s}}\right)
\end{aligned}
$$

Here $\sigma_{\alpha \beta}^{\star}$ denotes the surface tension of the unstrained reference state. This material model based on the Hencky finite-strain measure will be used to compute the apparent stress of synthetic drop shapes with modest deformations. However, the framework presented here can also be used with other material models. 


\section{Methodology for isotropic and anisotropic interfaces}

\subsection{Calculating synthetic drop shapes with isotropic surface tension}

Extending axisymmetric drop-shape analysis [5] to elastic interfaces has some inherent limitations [25]. Therefore, alternative approaches may be required. However these need to be tested just as well on the isotropic Young-Laplace differential equation. A spectral method [28] is well suited for smooth solutions and yields an order of convergence that is one order less then the number of unknowns used in the discretization. In spirit, the method is analogous to a fully implicit finite-difference method and formulates the linearized equations (2a,b) and eq.(5) with eq.(3a,b) as a matrix for the unknowns $\mathbf{r}, \mathbf{z}$ and $\boldsymbol{\psi}$. Every differential equation that is discretized on $N$ number or nodes results in an algebraic system with one equation for every discrete point. The forward problem, computing the drop shape from known parameters, results in a square matrix with as many equations as unknowns.

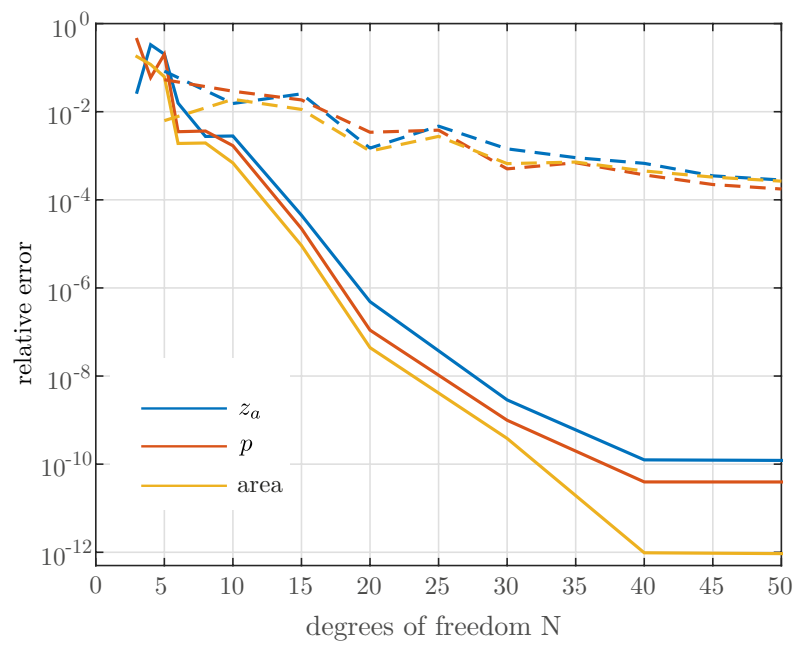

Figure 4: For $\sigma_{\alpha \beta}=30$ and $v=150$ the height of the relative error of the apex $z_{1}$, pressure $p$ and surface area $A$ of a drop are plotted against the degrees of freedom. The reference solution was obtained numerically with a more refined calculation $(N=100)$.

For a specific $\sigma_{\alpha \beta}$ and pressure $p$ the Young-Laplace equation with the geometric identities in $r, z$ and $\psi$ in eq. $(2 \mathrm{a}, \mathrm{b})$ results in $3 N$ equations with $3 N+1$ unknowns $r_{i}, z_{i}$ and $\psi_{i}$ and the unknown length of the domain $s$. The three first-order differential equations have as boundary condition $r_{1}=0, z_{N}=0$ and $\psi_{1}=0$. The missing equation is the additional boundary condition $r_{t}=r_{N}=1$ that imposes the needle radius and fixes the domain size $s$. A Newton scheme can be used to solve the system of non-linear equations iteratively. In order to assure that the method converges we give an example in figure 4 by computing a drop with $\sigma_{\alpha \beta}=30$ and volume $v=150$ and compare the drop height $\left|z_{a}\right|$, pressure $p$ and surface area $a$ for a changing number of unknowns to a highly refined computation in Figure 4 . For the spectral method (full line) one observes the characteristic exponential convergence with a straight line in a semilogarithmic representation, where due to the machine precision the accuracy is unchanged after $N=40$. For comparison a finite-difference scheme shows second order convergence (dashed line), where a 10 fold increase of unknowns leads to a 100 fold error reduction. Technical aspects of the method are presented in the Appendix B.

\subsection{Tensiometry of interfaces as a fully coupled inverse problem}

In the classical drop-shape analysis (DSA) and following the seminal work of Rotenberg [2], interfaces are numerically computed with an estimated surface tension (i.e. using a shooting method). The computed positions are then compared to experimentally sampled coordinates. An iterative procedure adapts the surface tension and pressure until the fitting error is minimized. A disadvantage of this method in generalizing this to (visco)elastic interfaces is that the drop-shape prediction and the problem parameters are iterated on separately, which is a particular issue when interfaces are elastic and more parameters need to be determined. For guessed values of the parameters the interface shape is computed, usually with a Runge-Kutta-like scheme, compared to the acquired shape, after which the parameter values are 
updated and a new guessed shape is computed until the error converges to a fixed value. Similarly, in methods such as the CPT, implicit assumptions about the homogeneity of the surface deformation are typically made, as the curvature is assumed to be uniform and known, and again the different aspects are decoupled. For these reasons often a hemispherical geometry is used. We will evaluate this assumption for elastic interfaces subject to deformation below.

To solve the non-linear fitting problem with coupled iterations, the shape and the parameters are more robustly determined at the same time. Solving this full inverse problem for a simple interface gives, in addition to the unknowns of the forward problem, two more unknowns, namely $\sigma_{\alpha \beta}$ and $p$, rendering the problem under-determined. However, additionally, $N$ equations imposing that the computed radius is equal to the sampled radius $r_{i}=\hat{R}_{i}$, are added, where $\hat{\mathbf{R}}$ are the experimentally sampled positions (see section 2.1). Hence, the discrete inverse problem is an overdetermined equation system with $4 N$ equations and $3 N+2$ unknowns. It is possible to fit the radius $\hat{\mathbf{R}}$ and height $\hat{\mathbf{Z}}$ obtained from the image but this has shown slightly lower convergence rates because the information of the locations $\hat{\mathbf{R}}$ and $\hat{\mathbf{Z}}$ is used to compute the arc-length $\hat{\mathbf{S}}$ and therefore $\hat{\mathbf{R}}$ contains the information of $\hat{\mathbf{Z}}$. Either way, because the problem is formulated in a single matrix updating the $\sigma_{\alpha \beta}$ and $p$ follows straight forward when solving the equation system and minimizing the residuals of the non-linear equations.

\subsection{Tensiometry of interfaces with a direct method}

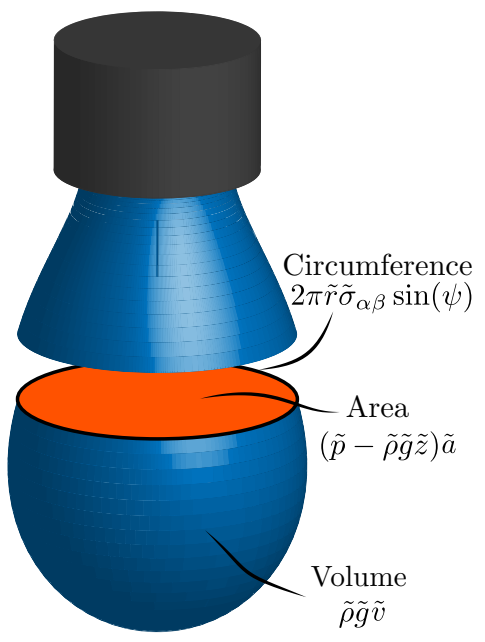

Figure 5: Illustration of the force balance with a virtual cut of the drop. The contributions are a body force from weight, a surface force due to pressure balanced by a line force due to surface tension.

The conceptually simplest method to obtain the isotropic surface tension from a pendant drop experiment relies on using a local force balance. Arabali and Peters [11] proposed a single local force balance, in combination with a pressure measurement to determine the surface tension. A virtual cut through the drop in the $r, \varphi$ plane allows to compare the gravitational body force and the pressure surface force to the surface stress along the circumference. Formulated in discrete variables this becomes:

$$
2 \pi r_{i} \sigma_{\alpha \beta} \sin \left(\psi_{i}\right)=\left(p-z_{i}\right) \pi r_{i}^{2}+v_{i} .
$$

The volume in the truncated region can be integrated according to:

$$
v_{i}=\int_{1}^{s_{i}} r^{2} \sin (\psi) d s .
$$

For isotropic interfaces it is actually not essential to measure the pressure because the unknown pressure and surface tension are recovered by performing at least two virtual cuts (for two values of $z$ ). The simplicity of implementing the equation without any need for an iterative procedure suggest this method to be fast and appealing at first sight. However, the precision of the method is expected to be lower than for an iterative parameter fitting because it involves a derivative of the shape coordinates $r, z$ in order to obtain $\psi$, which is prone to numerical error propagation, amplifying the unavoidable experimental noise in the images. The different methods will be compared below. 


\subsection{Drop shape and volume of an isotropic drop}

The precision of the pendant drop method to determine surface tension depends on the drop size, where larger volumes typically lead to higher accuracy. The largest stable drop volume before detachment is an important quantity. For our numerically generated droplets it can be estimated with the methods presented in the previous section. The drop shape is computed and its volume is increased iteratively until no stable solution can be obtained any more for a given $\sigma_{\alpha \beta}$ (the trends observed mimic what is observed experimentally). In the non-dimensional framework the volume of these drops depends on $\sigma_{\alpha \beta}$ and $p$. For the dimensional problem, changing the needle radius will affect the boundary condition at the needle tip, with radius $\tilde{r}_{t}$. For the non-dimensional problem, changing the needle radius leaves $r_{t}$ unchanged, for ideal wetting properties $r_{t}=r_{c}=1$, however the non-dimensional surface tension $\sigma_{\alpha \beta}$ will change because it depends on $r_{c}$. We will be interested in the largest drop volume that can be held on the needle for a given surface tension and compute numerically a master curve of volumes against $\sigma_{\alpha \beta}$ (the pressure $p$ is obtained from the maximization). As can be seen in Figure 6, in the limit of $\sigma_{\alpha \beta}>1$ (or Bond number $B o<1$ ) the volume is well approximated by $v=5 \sigma_{\alpha \beta}=5 / B o$, which agrees well with the low Bond number limit in the experimental work of Gunde et al. [33]. As can be seen in the inset, where $v / \sigma_{\alpha \beta}$ is plotted over $\sigma_{\alpha \beta}$, the proportionality between $v$ and $\sigma_{\alpha \beta}$ actually varies and attains a minimum at about 4.75 .

The dimensional volume is approximately given by:

$$
\tilde{v}_{\max } \approx 5 \sigma_{\alpha \beta} \tilde{r}_{c}^{3}=5 \frac{\tilde{r}_{c} \tilde{\sigma}_{\alpha \beta}}{\tilde{\rho} \tilde{g}} .
$$

In order to illustrate the change in volume when changing the capillary radius we take the quotient of two volumes with the same density and surface tension, one with a variable capillary radius and one for a radius $r_{c, 0}$, that is chosen to fulfill $\sigma_{\alpha \beta}=1, \tilde{r}_{c, 0}=\sqrt{\tilde{\sigma}_{\alpha \beta} / \tilde{\rho} \tilde{g}}$.

$$
\frac{\tilde{v}_{\max }}{\tilde{v}_{0}}=\frac{\sigma_{\alpha \beta} \tilde{r}_{c}^{3}}{1 \cdot \tilde{r}_{c, 0}^{3}}=\frac{\frac{\tilde{\sigma}_{\alpha \beta} \tilde{r}_{c}}{\rho g}}{\left(\frac{\sigma_{\alpha \beta}}{\rho g}\right)^{3 / 2}}=\sqrt{\frac{\tilde{\rho} \tilde{g} \tilde{r}_{c}^{2}}{\tilde{\sigma}_{\alpha \beta}}}=\frac{1}{\sqrt{\sigma_{\alpha \beta}}} .
$$

For increasing dimensional radii, $\tilde{r}_{c}$, the non-dimensional $\sigma_{\alpha \beta}$ decreases and the dimensional volume increases. Figure 6 shows the dimensional drop volume relative to the volume of a drop with the same

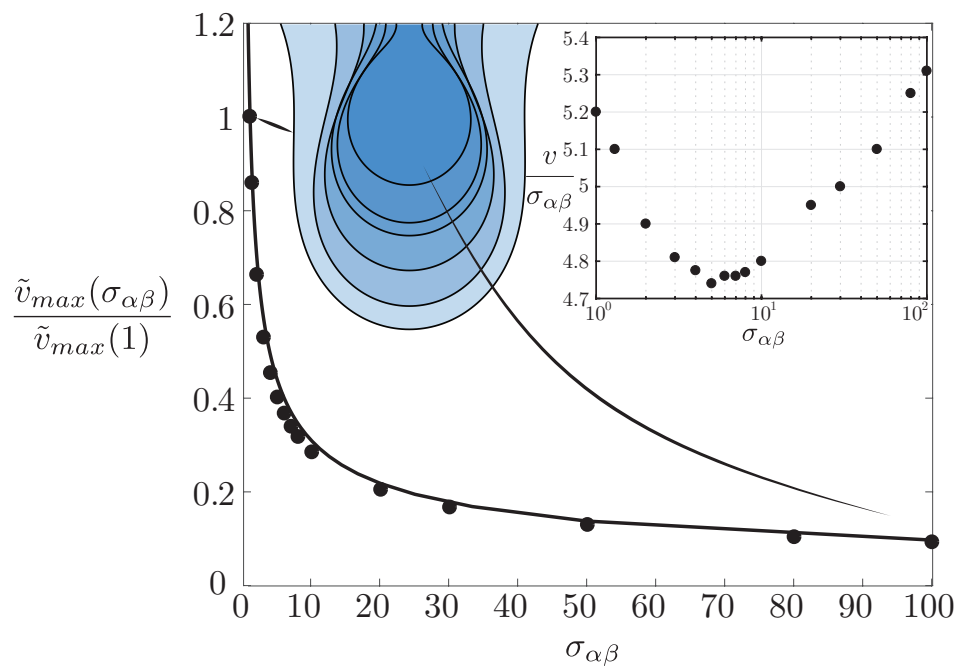

Figure 6: Volume ratio for changing needle radii, expressed by $\sigma_{\alpha \beta}$. Numerical values $(\bullet)$ and trend $v_{\max } / v_{0}=\sqrt{1 / \sigma_{\alpha \beta}}$ $(-)$ is shown. The inset shows that the dimensionless ratio is almost linearly dependent on $\sigma_{\alpha \beta}$. Drop images illustrate the shape for different $\sigma_{\alpha \beta}(=1,3,8,15,30 \& 100)$.

liquids but with a capillary radius such that $\sigma_{\alpha \beta}=1$. The inset shows the non-dimensional maximum volume against $\sigma_{\alpha \beta}$. Increasing the dimensional needle diameter decreases $\sigma_{\alpha \beta}$ and decreases the nondimensional volume. Smaller $\sigma_{\alpha \beta}$ augments the influence of the neck region, where in the case of elastic deformation the most anisotropic stress state is expected. 


\subsection{Computing synthetic drop shapes with anisotropic surface stresses}

When solving the drop interface with an elastic constitutive model the number of unknowns increases to $7 N\left(r, z, \psi, \sigma^{s}, \sigma^{r}, \lambda^{s}\right.$ and $\left.\lambda^{r}\right)$. The equations that be solved are the geometric identities that relate $r$ and $z$ to $\psi$ eq.(2a,b), the generalized YL eq.(6) together with eq.(7). Yet, there is need for a constitutive model such as eq.(11) and eq.(12) and at last the identity $\lambda^{r}=r_{i} / r_{i}^{\star}$, to give $7 N$ equations. These equations are subject to the boundary conditions $r_{1}=0, z_{N}=0, \psi_{1}=0$ and $\frac{d \sigma_{1}^{s}}{d s}=0$. The material model is not a differential equation and needs no boundary conditions, however, the last equation $\lambda_{1}^{r}=r_{1} / r_{1}^{\star}=0 / 0$ is singular at the apex. To remove this singularity it suffices to impose that at this position the strains in the different directions are equal $\lambda_{1}^{r}=\lambda_{1}^{s}$.

A closed system of $7 N$ equations is obtained, which allows to compute elastically deformed interfaces starting from an isotropic state that furnishes $r^{\star}\left(s^{\star}\right)$. An isotropic drop shape (the unstrained reference state) is calculated first, using a given surface tension $\sigma_{\alpha \beta}^{\star}$ and volume $v^{\star}$. The surface tension and the profile $r^{\star}\left(s^{\star}\right)$ serve as a reference state to compute the elastic drop shape, where we impose $K, G$ and either a volume $v$ or surface area $a$.

The arc-length $s$ of the deformed state is computed by integrating $\lambda^{s}$ and then all deformed variables are interpolated onto a unstretched grid with the new arc-length. Hereafter the synthetic drop shape in the strained and unstrained state are defined on their respective arc-length domain.

\subsection{Elastometry for elastic interfaces as an inverse problem}

Inverse fitting is achieved by creating an equation system with the additional unknowns to characterize the constitutive behavior of the interface. These are $E$ and $p$ with the isochoric neo-Hookean model [23] or $K, G$ and $p$ with the linear Hookean approach [25]. In these methods the isotropic surface tension is provided by an isotropic reference state. In order to close the system of equations the collocation of computed and sampled coordinates $r_{i}=\hat{R}_{i}$ is imposed. A complication is that the $r_{i}$ are typically sampled on a basis $s_{i}$ that does not reflect the fact that $\lambda^{s}$ actually links where $r_{i}$ and $r_{i}^{\star}$ have to be compared. The equations and the solution routines used in this work are summarized in Appendix C.

For small deformations $\lambda \sim 1$ the equation system becomes singular and this inherently leads to large errors. This is a fundamental problem of all shape fitting routines, in particular for experiments where one seeks to interrogate the linear viscoelastic response regime. It will also be a fundamental problem for analyzing oscillating drop experiments. Regardless even of the detection limits of the optical system, there will be the need for rather large deformations in all of these experiments. In practise a further complication is that an isotropic reference state has to be provided, as was pointed out by Danov et al. [26]. In experiments this is not always easy to guarantee that a structured interface is not stressed. For certain interfacial substances it is even desirable to pre-compress an interface to generate a sufficiently high surface coverage or due to diffusion or evaporation drop/bubble volumes may change. The pre-stress state is in direct conflict with the requirement of having an unstrained reference state for the above analysis to be valid and of practical use.

The fitting routines are non-linear equations and need an initial value, which will determine the convergence to some extent. For elastic interfaces, initial values for $K$ can, for example, be obtained from CPT, which does not require an initial guess. Then $G$ could be guessed with a few values between $K / 10 \ldots K$.

\subsection{Elastometry with a direct method}

Danov et al. [26] developed the Capillary Meniscus Dynamometry (CMD) method for dealing with elastic interfaces, which consists of computing a non-uniform $\sigma^{s}(s)$ with eq.(13), generalized to:

$$
2 \pi r_{i} \sigma^{s} \sin \left(\psi_{i}\right)=\left(p-z_{i}\right) \pi r_{i}^{2}+v_{i} .
$$

Thereafter $\sigma^{r}(s)$ is obtained from eq.(7) or equivalently from:

$$
\sigma^{r}=\frac{p-z-\sigma^{s} \kappa^{s}}{\kappa^{r}}
$$


In order to compute $\sigma^{s}$ one needs to provide the pressure $p$ by a measurement with a pressure transducer that allows to determine the pressure inside the capillary. As a consequence of solving a closed system of equations (eq.(17)) that is not overdetermined, errors are not damped. In fact, the equation systems is rather unstable against perturbation of the coordinates. Consequently, the computed stress profiles can display spurious oscillations, which are due to noise in the acquired data and amplified by applying a derivative to the data. This is partially the case when computing $\sigma^{s}$, which depends on $\sin (\psi)=d z / d s$, and much worse for $\sigma^{r}$ (eq. (7)), which depends on the derivative of $\sigma^{s}$ eq.(17) or a double derivative of $r$ in the curvature eq.(18). These difficulties become apparent when computing a stress of a simple interface that is constant and isotropic, because the oscillations are practically unavoidable. Mainly for this purpose we developed the Chebyshev filtering explained in section 2.1.

Since we are interested in material parameters it is necessary to go beyond the CMD method and include a constitutive relation to compute the compression modulus $K$ and shear modulus $G$ from the obtained stress profiles. If the shear modulus is negligible the simplest method, analog to assumptions typically used in the analysis of the capillary pressure tensiometer (CPT) [34], is to compare the change in surface tension at the apex to a change in area as in eq.(9). This is justified as long as the deformation is homogeneous, $\lambda^{s} \lambda^{r}=$ constant. But it does not imply $\lambda^{s}=\lambda^{r}$, which was for example suggested by Ferri et al. [24]. In order to obtain both $K$ and $G$ an inverse problem has to be solved, made up of 420 the equations for the stress-strain equation in $s$ and $r$ direction (eq.(11) and 12). The $2 N$ equations determine $N+2$ parameters, which are $\lambda^{s}, K$ and $G$. All other quantities $r, z, \psi, \sigma^{s}, \sigma^{r}$ and $r^{\star}$ are given from shape sampling and CMD. We hereafter refer to this method as stress-fitting elastometry (SFE)

An important improvement of the direct method compared to the shape fitting methods is that it can be generalized to compare two elastically deformed states and does not require an isotropic reference state to start the experiments. This addresses the main drawback of the existing elastometric approaches as pointed out by Danov et al. [26] and makes the method possibly more versatile and easier to use. Two discrete equation systems are obtained by subtracting the stress-strain relations of two interfaces, in which case $\sigma_{\alpha \beta}^{\star}$ is eliminated. Due to the nature of the Hencky strain the dependence on the isotropic reference state also cancels out,

$$
\ln \left(\lambda_{I I}^{s}\right)-\ln \left(\lambda_{I}^{s}\right)=\ln \left(\frac{\lambda_{I I}^{s}}{\lambda_{I}^{s}}\right)=\ln \left(\frac{d s_{I I}}{d s^{\star}} \frac{d s^{\star}}{d s_{I}}\right)=\ln \left(\frac{d s_{I I}}{d s_{I}}\right)=\ln \left(\lambda^{s}\right) .
$$

Consequently $\lambda^{s}=d s_{I I} / d s_{I}$ and analogous $\lambda^{r}=r^{I I} / r^{I}$. This changes eq.(11) and eq.(12) into:

$$
\begin{aligned}
\sigma_{I I}^{s}-\sigma_{I}^{s} & =K \ln \left(\lambda^{s} \lambda^{r}\right)+G \ln \left(\frac{\lambda^{s}}{\lambda^{r}}\right), \\
\sigma_{I I}^{r}-\sigma_{I}^{r} & =K \ln \left(\lambda^{s} \lambda^{r}\right)+G \ln \left(\frac{\lambda^{r}}{\lambda^{s}}\right) .
\end{aligned}
$$

The strain $\lambda$ used in these equations does not refer to an initial state but measures the relative deformation between state $I$ and state $I I$. Just as in the previous section $\lambda^{s}$ controls where $r_{I}$ is evaluated when compared to $r_{I I}$ for the evaluation of $\lambda^{r}$. Additionally also $\sigma_{I}^{s}$ and $\sigma_{I}^{r}$ have to be interpolated to the right location.

It is instructive to reformulate the above equations in order to single out the influence of $K$ and $G$ :

$$
\begin{aligned}
\sigma_{I I}^{s}-\sigma_{I}^{s}+\sigma_{I I}^{r}-\sigma_{I}^{r} & =2 K \ln \left(\lambda^{s} \lambda^{r}\right), \\
\sigma_{I I}^{s}-\sigma_{I}^{s}-\sigma_{I I}^{r}+\sigma_{I}^{r} & =2 G \ln \left(\frac{\lambda^{s}}{\lambda^{r}}\right) .
\end{aligned}
$$

The first equation shows that the sum of the stress difference determines $K$. The largest effect of the compressional/dilatational contributions can be found near the apex, where the stress state is isotropic. The shear modulus is determined by the difference of the meridional and hoop stress difference. At the apex this equation is singular because for isotropic stresses the left and right hand side of the equation are zero, independent of the value of $G$. The strongest contribution is expected near the neck of the drop, i.e. near the capillary, which leads to an anisotropic deformation as the capillary itself is undeformable and with the contact line pinning of the interface this leads to $\lambda^{r}=1$. 
These equations have important consequences for experiments and these implications go beyond what is discussed here for static drops or step strain experiments, but also hold for oscillatory drops. For pure compression/dilation rheology it is advantageous to work with interfaces of high $\sigma_{\alpha \beta}^{\star}$ and large volumes, minimizing the effect of the neck region, i.e. as the inset in figure 6 having the drop or bubble outside of the capillary (which is not how CPT measurements are currently performed). When the ratio of drop volume over capillary radius is large $\left(v / r_{t}^{3} \gg 1\right)$, the anisotropic region near the capillary shows a less pronounced influence, which improves the precision of $K$ but precludes any information on $G$. If one wants to obtain the shear properties at the same time, one needs to have a neck region. The surface elements close to the capillary will show non-uniform stretching. This also implies that for the hemispherical geometry used in the CPT technique does not ensure homogeneous deformations. A near spherical geometry with where the needle is small compared to the drop features would work as we will demonstrate in section 4.4. We will quantify the deviations in homogeneous deformation below. Although the analysis above uses the Hencky strain measure, the method works basically also for other constitutive models where $\lambda$ relates to the stretching of one deformed state in comparison to the other deformed state. Other strain measures or material models led to a slight deviations for synthetic-numeric fitting when the reference state is shifted away from the reference state.

\section{Evaluation of the methods to obtain the surface stress}

In the following, the iterative inverse method (global fitting) and the direct method (local force balance) will first be compared in their ability to measure the interfacial tension for simple interfaces, to establish the base case. Then their suitability for obtaining elastic stresses and constitutive parameters will be discussed. Within our analysis of deformed interfaces we use moderate moduli that are of a similar magnitude as the surface tension in combination with moderate compression ratios. Consequently the interfaces remained always in a tensile state. Wrinkling analysis and determination of the bending modulus is a merit of Knoche et al. [25] that we did not try to reproduce since we were mainly interested in small deformations for the sake of a linear response.

\subsection{Simple interfaces : interfacial tension}

For pendant drops with an isotropic surface tension, the inherent accuracy of the fitting method depends on the extent of drop deformation. For small or near spherical drops with a high $\sigma_{\alpha \beta}$, or low Bond number, the curvature gradient is very small and, therefore, prone to disturbance by noise in the data. Several authors have characterized the "quality" of a drop shape for instance with a shape parameter $P$ [35] or a Worthington number Wo [7].

The shape parameter $P$ was defined by Neumann and coworkers and revised several times [35]. In its initial definition, it compared the area of a circle that is fitted at the drop apex to the area of the drop projected onto the $(r, z)$ plane. A revision from the same group [8] redefined $P$ as root-mean-square of the deviation between the drop interface and the periphery of the best fitting circle whose center is located at $r_{0}, z_{0}$ and whose radius is $R_{0}$.

$$
P=\frac{1}{R_{0}} \sqrt{\frac{1}{N} \sum_{i=1}^{N}\left(\sqrt{\left(r_{i}-r_{0}\right)^{2}+\left(z_{i}-z_{0}\right)^{2}}-R_{0}\right)^{2}} .
$$

The accuracy increases with $P$, when the shape deviates from the spherical shape, and consistent measurements of surface tension are reported for $P>0.04$.

Alternatively, Berry et al. [7] presented a scaling of the error in terms of a rescaled Bond number, which they named the Worthington number:

$$
W o=B o \frac{\tilde{v}}{2 \pi \tilde{r}_{c}^{3}}=\frac{v}{2 \pi \sigma_{\alpha \beta}}=\frac{\tilde{\rho} \tilde{g} \tilde{v}}{2 \pi \tilde{\sigma}_{\alpha \beta} \tilde{r}_{c}} .
$$

The length dependence in the Bond number $\tilde{r}_{c}^{2}$ is substituted by $\frac{\tilde{v}}{2 \pi \tilde{r}_{c}}$, the drop volume over the needle circumference $2 \pi \tilde{r}_{c}$. The accuracy also increases with $W_{o}$, with $W_{o}>0.5$ typically leading to good precision. The Worthington number is a good a priori indicator, because it allows, based on an estimate 
of the surface tension, to design the appropriate experimental conditions by adapting the capillary radius and the required drop volume. The shape parameter $P$ is suitable as an a posteriori check of the obtained precision because it requires a drop shape.

As can be seen in the inset in Figure 6 for small $\sigma_{\alpha \beta}$ the needle gains an increasing influence on the drop shape with the consequence that for any effect that is not axisymmetric causes a disturbance that makes the drop geometry deviate from the axisymmetric Young-Laplace shape. Possible reasons are needle misalignment with the $z$ axis, needle tip roughness or variations in wettability. These disturbances will decay away from the needle tip with a typical length scale proportional to the needle diameter, hence large $\sigma_{\alpha \beta}$, with a smaller neck region, reduce the effect of needle induced imperfections.

\subsection{Comparing fitting and direct shape analysis for simple interfaces}

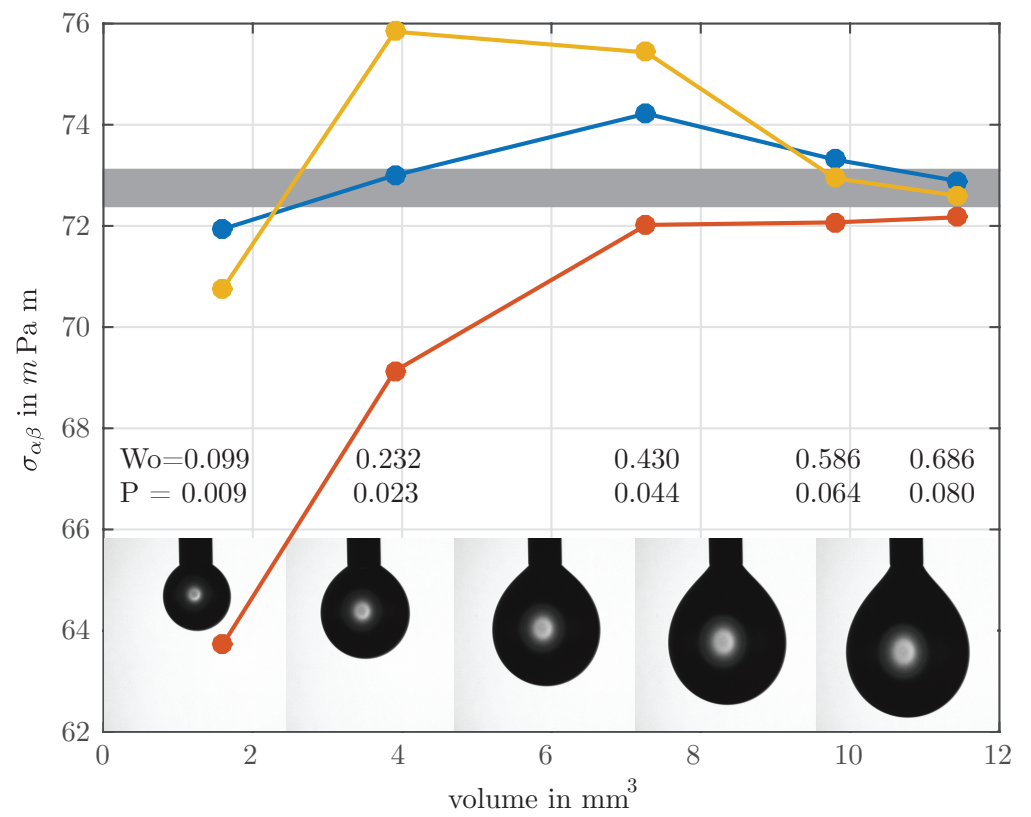

Figure 7: Evaluation of methods to determine surface tension for a water drop in air for different volumes using a classical shape fitting algorithm (DSA, yellow) [7], compared with a global fitting based on Chebyshev interpolation (DSA, blue) to the use of a local force balance (red). The Worthington number Wo and the $\mathrm{P}$ shape parameter are given with each image.

Before turning our attention to elastic interfaces, the precision of the classical shape fitting method, the modified shape fitting algorithm using the Chebyshev transform and the local force balance method is compared. To this end, the reference images by Berry et al. [7] have been used. The density difference is $1000 \mathrm{~kg} / \mathrm{m}^{3}$ and the needle size was $0.72 \mathrm{~mm}$. The theoretical surface tension at the temperature of the experiments is expected to be $\tilde{\sigma}_{\alpha \beta}=72.75 \mathrm{mPam}$ [7]. The resulting expected non-dimensional value of $\sigma_{\alpha \beta}$ is 56.5. Frame 1, the largest drop, has a volume of about $11 \mathrm{~mm}^{3}$ corresponding to a Worthington number of $W_{0}=0.69$, which should yield accurate measurements $\left(W_{O}>0.5\right)$. Frame 5 , the smallest drop, has a volume of $1.6 \mathrm{~mm}^{3}$ and Wo $=0.01$, which is expected to give deviations in the order of $5 \%$.

Figure 7 shows the image series and compares results for the different methods with the expected surface tension. The DSA implementation in opendrop by Berry et al. (yellow) and the inverse fitting methods based on the Chebyshev transform perform similarly well. The direct tensiometry method, which will be amenable to the most straightforward generalization with elastic interfaces, is less robust, and shows a larger error for small drops. Also given is the shape parameter $P$ and the Worthington number $W_{O}$. Only the two largest drops show $P$ and $W_{O}$ above the empirical threshold that suggest confident results. It is shown that all three methods converge to within sufficient accuracy. Figure 7 confirms that for accurate determination of the surface tension without a pressure measurement the curvature gradient needs to be ample. 


\subsection{Evaluating the validity of the Young-Laplace equation}

Even when pendant drops experiments are carried out under the right experimental conditions for $P$ and $W o$, it has been observed that there can be a systematic fit error of the Young Laplace equation when the interfaces become complex, for example, as they undergo a transition from fluid to elastic solid films in the case of hydrophobins [18]. However, the use of the fit error of the Laplace equation to characterize elastic behavior was rightfully critiqued by Danov et al. [26]. A more obvious test to see if the drop obeys the Young-Laplace equation is to plot the curvature as a function of height $z$, and verify if it follows a straight line. However, in practice this result is not easily obtained as the meridional curvature contains a double derivative of the variables $r, z$ and is therefore, prone to noise propagation.
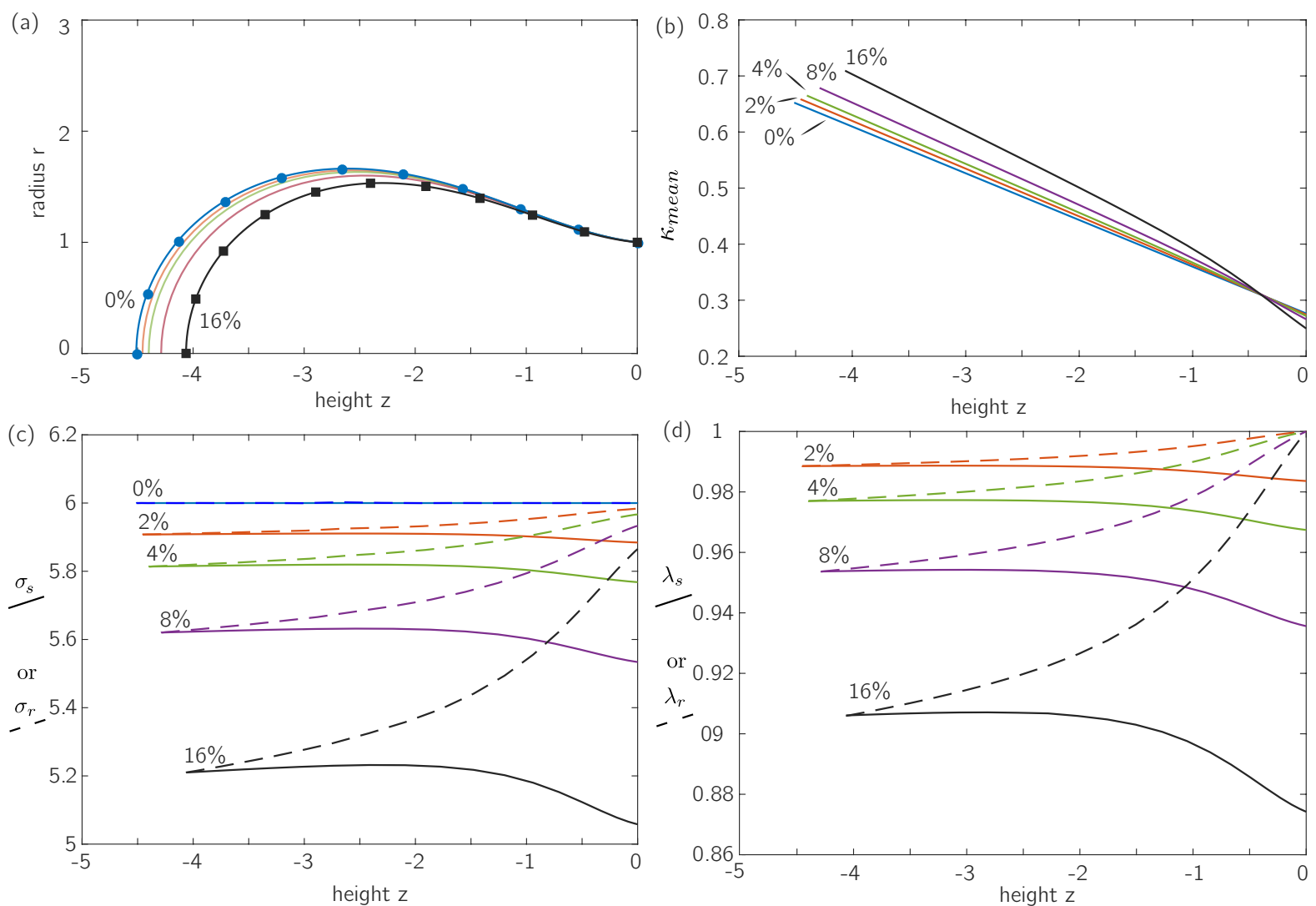

Figure 8: Synthetic drop images, calculated with with $\sigma_{\alpha \beta}^{\star}=6, v^{\star}=27, K=4$ and $G=3$ for different area compressions $(a / a \star=0,2,4,8$ and $16 \%$ compression) (a) Drop shapes (b) Curvature profiles, (c) stress profiles and (d) stretches for the deformed states.

As an illustrative example an example drop shape was calculated with the initial surface tension $\sigma_{\alpha \beta}^{\star}=6$ and the initial volume $v=27$, which shows a moderate neck region. The elastic moduli of the interface were chosen to be $K=4$ and $G=3$, where $K$ for is lower than one expects for the highly elastic colloidal monolayers or protein assemblies and $G$ is smaller than $K$ so that $\nu>0$. The drop is compressed in steps by surface area fractions of $2,4,8$ and $16 \%$ and the shapes plotted in (a) with markers on the initial and final state $(16 \%)$. In Figure 8 the shape, curvature, as well as the stretches and stresses are shown. Figure 8 (b) shows that the curvature at the drop apex increases logically with increasing compression. Yet near the neck it actually decreases leading to a curvature profile in $z$ that is no longer linear. The stretches (d) show meridional and circumferential compression at the drop apex and an uncompressed circumference which is imposed by contact line pinning at the capillary $(\mathrm{z}=0)$ that is compensated by an increased meridional compression. From the isotropic compression at the apex follows an isotropic stress at the apex whereas the stresses at the capillary are anisotropic with a lower stress for the meridional contribution due to the larger compression (c).

The quantities plotted in figure 8 can be obtained from experimental images as well. For a validation whether the drop has a simple interface, evaluation of the curvature (fig. 8 (b)) is actually not a 
convenient tool. Even in the absence of noise from fitting the difference of a deviation from a straight line becomes only perceptible for deformations in the order of $10 \%$. Danov et al. proposed to use ${ }_{535}$ the CMD method (sec. 3.7), where a pressure measurement is carried out, to evaluate if the stresses are homogeneous. Indeed, the stress is more sensitive (shown in Figure $8 \mathrm{c}$ ) than the curvature. The presence of extra stresses reveals itself quite readily by the higher than expected pressure upon dilatation.

Figure 8 shows that when the shear modulus $G$ is non-zero, the stress is anisotropic and consequently the area change $\lambda^{s} \lambda^{r}$ is not uniform along $s$. Stress anisotropy will primarily occur in and around the neck as the pinning of the contact line near the capillary hinders radial compression and fixes the length in one direction. This will entail shape changes when the drop is deformed for surface elements near the capillary. This implies, for example, that one cannot always use a priori the assumption of homogeneous deformation of a hemisphere, as in eq.(9) as for example done by Kotula and Anna [34]. The stress, for example, at the drop apex, can not be related to a given compression. Only with a stress-strain relation can $\lambda^{s}$ and, consequently, $\lambda^{r}$ be resolved or other configurations need to be chosen which minimize the effect of the deformations near the neck, having for example the drop/bubble outside of the capillary. As already mentioned, at the apex, due to symmetry, the stress state is isotropic and only subject to compression. At the apex the total area change of the whole drop interface might still be a good approximation for the local compression. The surface stresses can be obtained from DSA, $\mathrm{CPT}$ or CMD. This allows to infer the dilatation modulus $K$. Information about the shear modulus $G$ requires anisotropic deformation and is, therefore, localized near the needle.

\subsection{Evaluation of isotropic methods used in elastometry}

In the following we will first evaluate the errors made by neglecting the deviatoric stresses. The first method, which is currently used in all commercial instruments, consists in fitting the Young-Laplace equation to the drop shapes, by classic isotropic tensiometry (DSA) or the so-called capillary-pressure tensiometry (CPT). The DSA method is a pure fitting approach that solves the Young-Laplace equation for both the surface tension and capillary pressure, whereas the CPT method solves the Young-Laplace equation only for the surface tension with an independently obtained pressure. The compression is obtained from eq.(9), which we reformulate for this purpose to yield an "effective" compression modulus, which is what would be obtained in experiments:

$$
K^{f i t}=\frac{\sigma_{I I}-\sigma_{I}}{\ln \left(a_{I I} / a_{I}\right)} .
$$

Comparing this effective value of $K^{\text {fit }}$ with the known $K$ (used to calculate the shapes) defines a relative error. This error has been evaluated for the different methods, using certain values of $\sigma_{\alpha \beta}^{\star}$ with varying $K$ or $G$. The volume was kept at $v=4.5 \sigma_{\alpha \beta}^{\star}$, since one is mainly interested in compression experiments where the initial volume should be maximal including safety margin for stability. The moduli are normalized by $\sigma_{\alpha \beta}^{\star}$ of the initial state in order to qualify the initial shape, in particular with respect to the importance of the neck region. $K / \sigma_{\alpha \beta}^{\star}$ has been varied in order to investigate the effect of increasing elastic contributions and then the ratio $G / K$ is varied in order to investigate the effect of deviatoric stresses. In experiments $\sigma_{\alpha \beta}^{\star}$ can be varied for given materials by changing the capillary diameter.

The modulus has been obtained by fitting the synthetic-numeric shapes, so no sampling discrepancies or noise are present, deviations are only due to inherent methodological short comings. Finite-strain deformations were performed ( 1 to $4 \%$ ) and the modulus extrapolated to zero strain. The surface tension of the initial state is assumed to be known or determined from an independent experiment of the undeformed reference state. Figure 9 shows data for different ratios of $K / \sigma_{\alpha \beta}^{\star}$ and with $G=K / 2$. With the DSA method, the errors are even more than a $100 \%$ for small values of $K$. It is clear that using the DSA method to obtain an effective $K$ value is not to be recommended, even when $K>\sigma_{\alpha \beta}^{\star}$. But even for the CPT method the errors are substantial at small values of $\sigma_{\alpha \beta}^{\star}$ because $K$ is difficult to detect and the influence of $G$ is not negligible. Acceptable errors are obtained when $\sigma_{\alpha \beta}^{\star}$ is sufficiently large, such that the effect of the neck region is limited, and reasonable values for $\mathrm{K}$ are obtained. This implies that experiments should be performed for such combinations of the capillary diameter and the surface tension that the drop or bubble is outside of the capillary, hence not using the hemispherical geometry that is now usually selected [34]. In that case, the pinning of the contact line at the hemisphere causes strong anisotropy of the deformation of the fluid elements next to the contact line, causing deviatoric stresses. In Figure 9(b) drop shape images illustrates qualitatively the size of the neck region. The non-dimensional surface tension $\sigma_{\alpha \beta}^{\star}$ can be adjusted with the needle radius, while the ratios $K / \sigma_{\alpha \beta}^{\star}$ and 

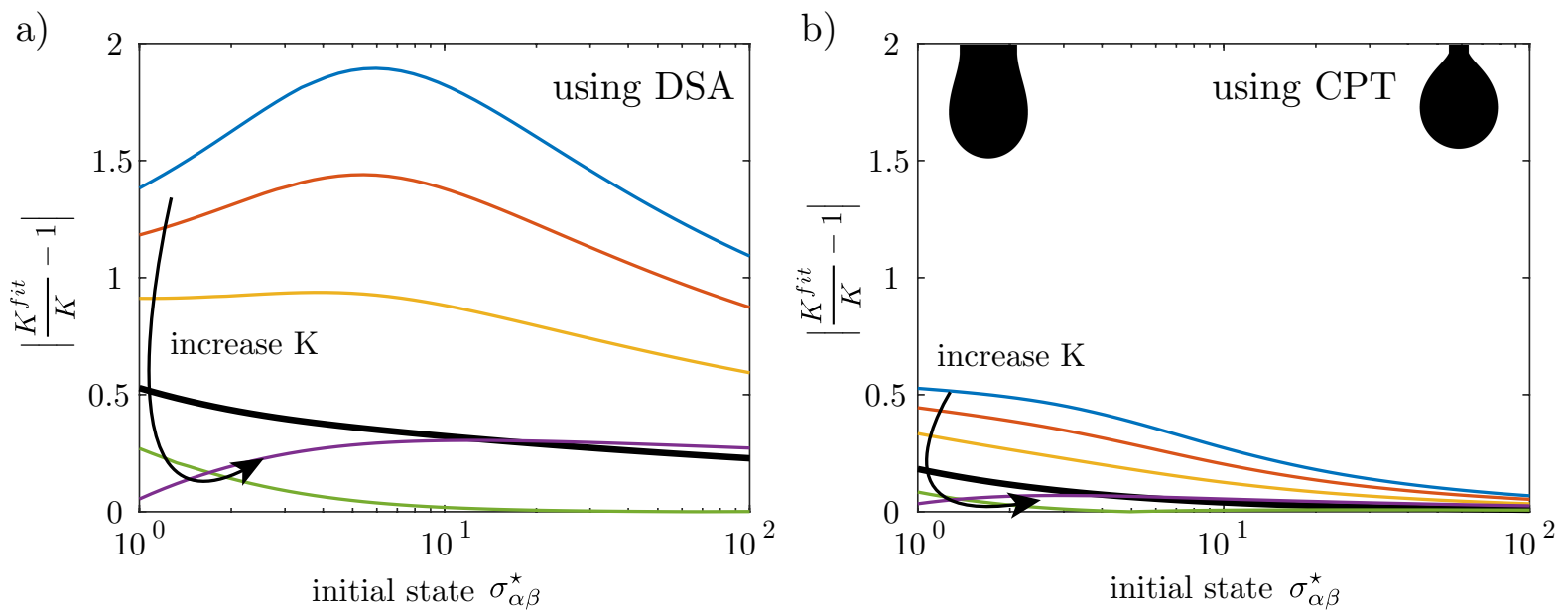

Figure 9: Relative error made when evaluating the compression modulus $K$, obtained using drop shape analysis (DSA, (a)) and capillary pressure tensiometry (CPT, (b)) as a function of dimensionless surface tension for $G=K / 2$, a strain of $4 \%$ and different values of $K\left(K=\sigma_{\alpha \beta}^{\star} / 10\right.$ blue, $K=\sigma_{\alpha \beta}^{\star} / 4$ red, $K=\sigma_{\alpha \beta}^{\star} / 2$ yellow, $K=\sigma_{\alpha \beta}^{\star}$ black, $K=1.5 \sigma_{\alpha \beta}^{\star}$ green and $K=2.5 \sigma_{\alpha \beta}^{\star}$ violet).

$G / K$ are fixed by the material properties.

a)

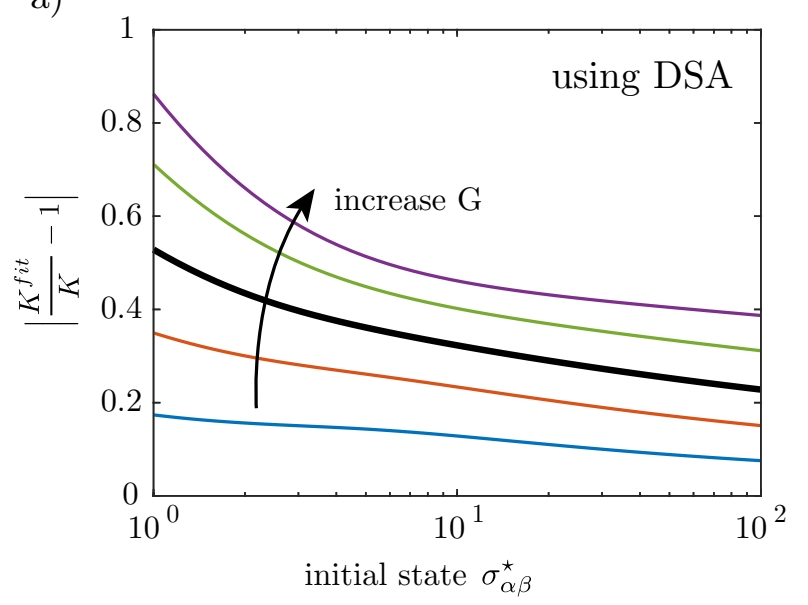

b)

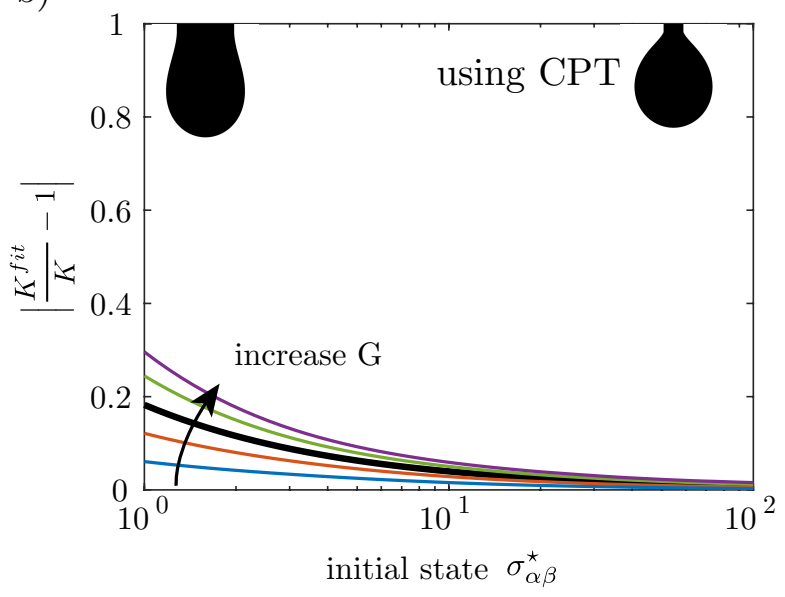

Figure 10: Relative error made when evaluating $K$ with the (a) the DSA method for different $\sigma_{\alpha \beta}^{\star}$, and (b) CPT method. Parameters : $K=\sigma_{\alpha \beta}^{\star}$ and $G=K / 10$ blue, $G=K / 4$ red, $G=K / 2$ black, $G=K$ green and $G=2 K$ violet.

In Figure 10 the effect of varying the shear modulus $G$ on the error on the "effective" compression modulus is shown. In congruence with the results above, larger values of $G$ lead to increased errors in (a) and (b), which assume an absence of deviatoric stresses. For a facilitated comparison figure (a) and (b) have been plotted to the same scale. For larger values of $\sigma_{\alpha \beta}^{\star}$, the influence of shear stresses becomes smaller as the neck region adjacent to the capillary shrinks. It has been suggested that the use of microgravity minimizes the effect of deviatoric stresses, because the internal pressure is constant and, consequently, $\lambda^{s}=\lambda^{r}$ [24]. Only with a uniform surface tension does a constant pressure difference leads to a constant curvature. But the analysis shows that the strains are not equal and, therefore, a non-zero shear modulus $G$ will lead to non-uniform surface stresses. Similarly, microtensiometry using small hemispherical caps has been advocated as a purely dilatational deformation, as the principal radii are constant [34]. In this case, the strains at the drop apex are indeed equal to one another, but the hoop strain $\lambda^{r}$ at the capillary is different, as a uniform deformation is hindered by the capillary geometry (and hence $\lambda^{r}=$ zero at the capillary), whereas the meridional strain $\lambda^{s}$ is not, which, induces shape changes and results in an influence of $G$. For the deformation to be dominated by dilation, it is advantageous to 
use a geometry such that the value of $\sigma_{\alpha \beta}^{\star}$ is large.

\subsection{Evaluation of methods used to determine the surface stresses}
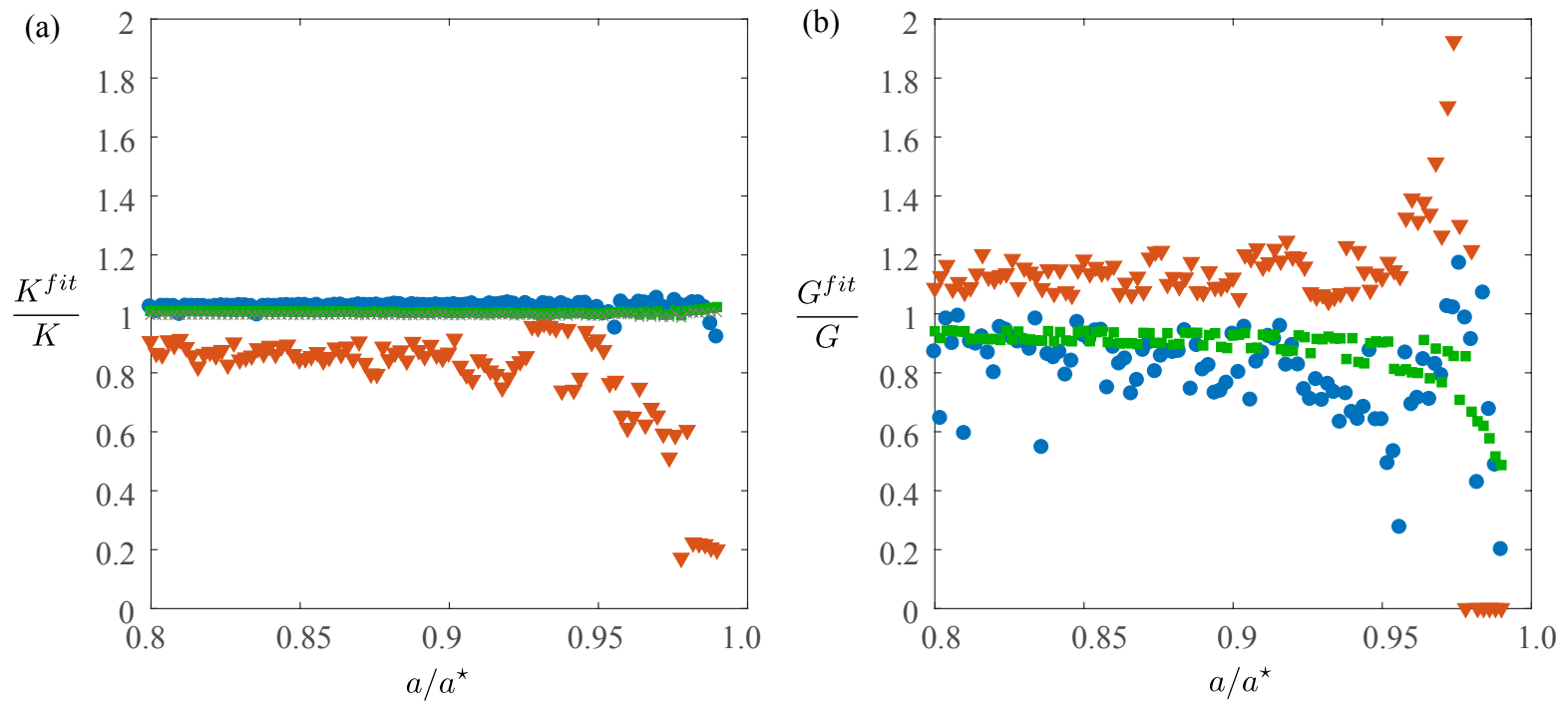

Figure 11: Relative errors on (a) $K$ and (b) $G$ with the parameters obtained from the analysis of computer generated shapes. Parameters $\sigma_{\alpha \beta}^{\star}=6, K=4$ and $G=3$. Shown are the relative errors against the interface compression: Stress-fitting elastometry (blue), drop shape fitting elastometry (red), pressure-assisted elastometry (green) and on (a) CPT (grey).

Three methods are compared here for their capability to retrieve material parameters. First the stress-fitting elastometry (SFE) presented in section 3.7, where a direct approach is used to compute the interfacial stresses and strain fitting is performed based on these computed stresses and the sampled geometry. Second and third are the shape fitting methods, the full inverse drop-shape fitting elastomery analogue to Knoche et al. [25], but using a pseudo-linear Hookean model for a fair comparison and pressure-assisted elastometry, which complements shape fitting with a pressure measurement. When appropriate these methods will be compared to the CPT method when the sole purpose is to retrieve the compression modulus. Because of the parameter space of five parameters that describe the synthetic drop shape, $\sigma_{\alpha \beta}^{\star}, v^{\star}, K, G$ and $a / a^{\star}$, we will pick out an example configuration and show how the different methods behave for this case. In the latter part we will explore a wider parameter space. The model interface is generated with the same parameter set presented in section 4.3, with initial surface tension $\sigma_{\alpha \beta}^{\star}=6$, initial volume $v^{\star}=27$ and elastic moduli $K=2 / 3 \sigma_{\alpha \beta}^{\star}=4$ and $G=3 / 4 K=3$. The applied deformation between the reference state and the deformed state will be $5 \%$ in area compression if not specified otherwise. Appendix G (figure F.17) shows that all methods used here converge for 30 point with the exponential convergence due to the accuracy of the spectral method. The sampling of the shape with Chebyshev filtering and the effect of the highest order of the Chebyshev polynomials for the different methods is also demonstrated in the appendix in Figure F.18

Any shape-fitting method suffers from singular behavior when the strains are small, as the stressstrain relations give values close to zero. With the Knoche method [25] the use of infinitesimal strain measures requires small compressions, whereas precision favors large compressions. Figure 11 shows how the fitted moduli values show strong oscillations when they are determined at small compressions. Even in the close to ideal case of synthetic-image fitting, still a compression of at least $5 \%$ is needed to achieve a reasonable accuracy for $K, G$. The stress-fitting elastometry (SFE) method, or methods that rely on pressure information (CPT, pressure-assisted elastometry), do not suffer as much from these disadvantages, at least as far as the determination of $K$ is concerned. The determination of the shear modulus is more prone to error.

Figure 12 and 13 show the variation of the expected moduli when $K$ and $G$ are varied for a typical choice of drop parameters (again the surface tension is assumed to be known or determined separately). From Figure 12 it can be concluded that the drop-shape fitting elastometry results in large relative 

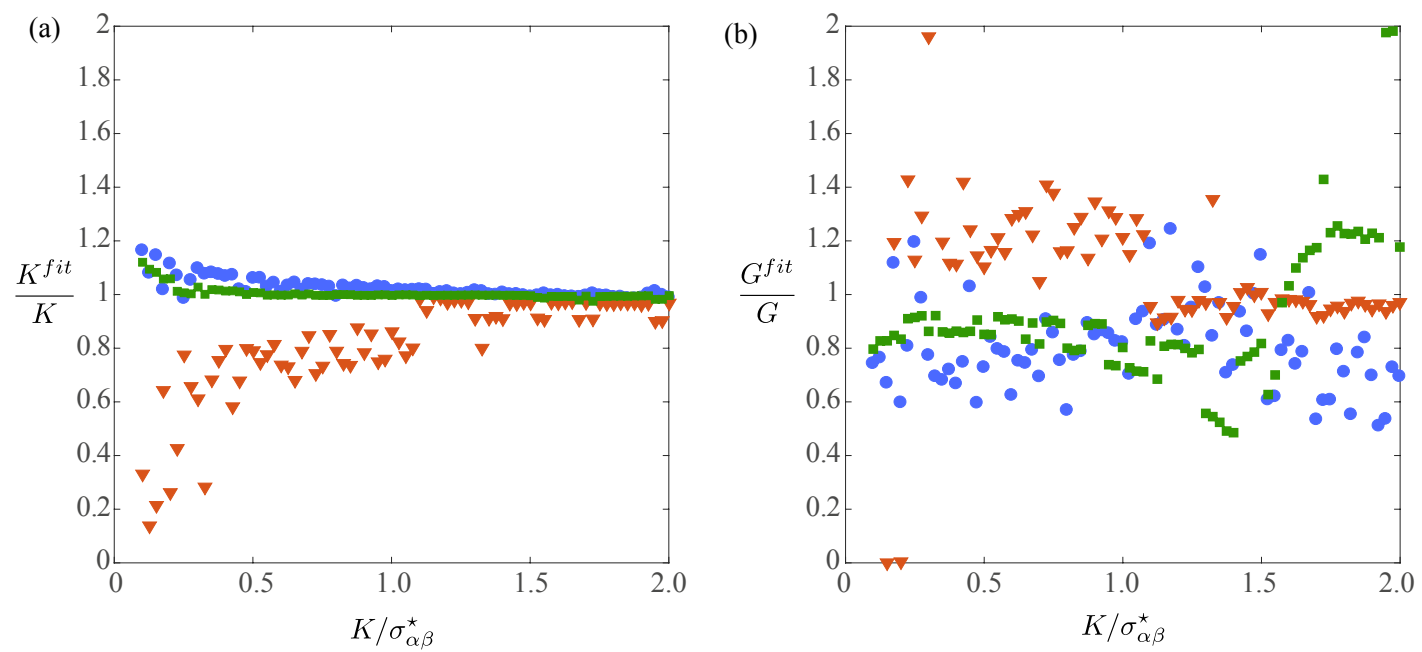

Figure 12: Sensitivity of the different methods to the relative magnitude of $K$. Fitted $K$ and $G$ values versus $K / \sigma_{\alpha \beta}^{\star}$ : Stress-fitting elastometry (blue), drop shape fitting elastometry (red) and pressure-assisted elastometry (green). $\sigma_{\alpha \beta}^{\star}=6$ and $G=3 / 4 K, v^{\star}=4.5 \sigma_{\alpha \beta}^{\star}$ and $5 \%$ area compression.
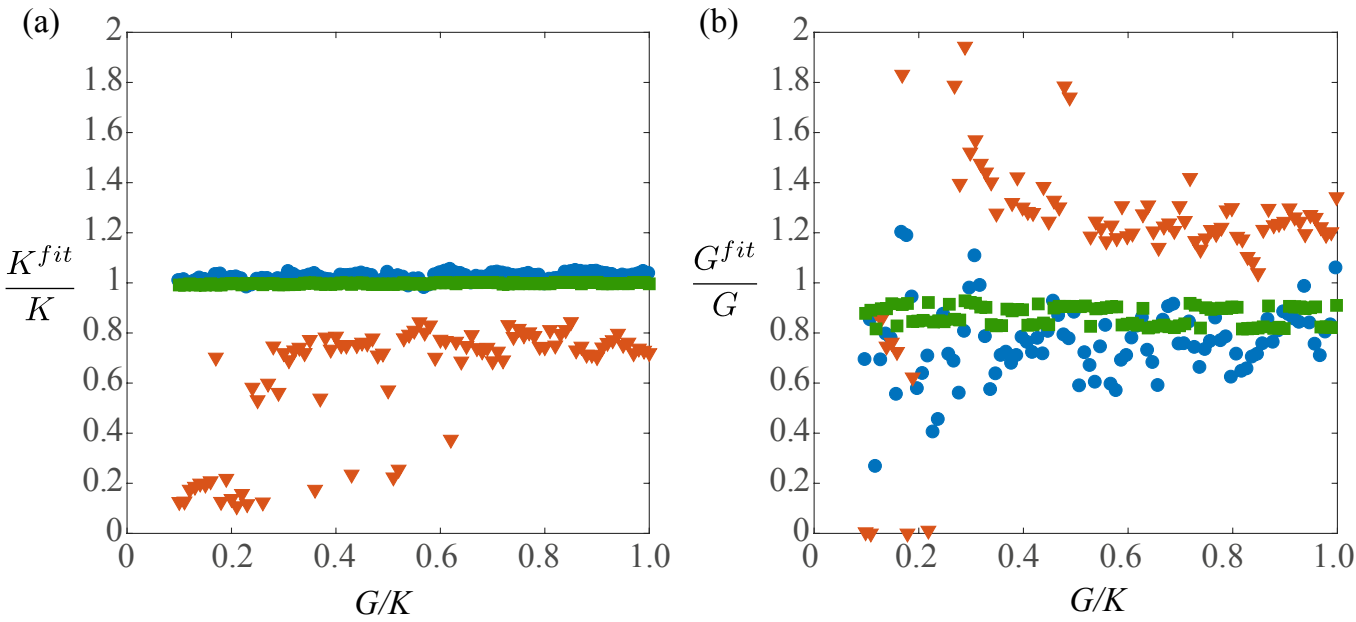

Figure 13: Sensitivity of the different methods to the relative magnitude of $G$. Fitted $K$ and $G$ values versus $G / \sigma_{\alpha \beta}^{\star}$ : Stress-fitting elastometry (blue), drop shape fitting elastometry (red) and pressure-assisted elastometry (green). $\sigma_{\alpha \beta}^{\star}=6$ and $G=3 / 4 K, v^{\star}=4.5 \sigma_{\alpha \beta}^{\star}$ and $5 \%$ area compression.

errors of $K^{\text {fit }}$ relative to $K$, for small values of $K$ relative to $\sigma_{\alpha \beta}^{\star}$. This is in contrast to pressure-assisted elastometry and stress-fitting elastometry that work well in this limit. As the values of $K$ are increased relative to $\sigma_{\alpha \beta}^{\star}$, all methods work well. Figure 13 shows that the noise on the shear modulus $G^{f i t}$ is higher, and this noise tends to become more prominent as the magnitude of $K$ is larger. It can be concluded that whereas shape fitting works very well for surface tension (see section 3.1), it is not a very robust method for the case of elastic interfaces, unless the pressure is also provided. The stress-fitting method, although noisy, works quite well. The shape-fitting methods do require that the initial reference state is isotropic.

\subsection{Robustness of the different methods in relation to experimental conditions}

In the preceding evaluations we have seen that the accuracy that can be obtained depends on the validity of the material model or strain measure, the sampling quality of the interface and, if applicable, on the precise determination of the pressure and the initial state. With a given setup and under the most careful handling, the user has still one degree of freedom to exploit, which is the choice of the capillary diameter. In Figure 14 we evaluate the expected values for $K^{f i t}$ and $G^{f i t}$ when changing the non-dimensional surface tension $\sigma_{\alpha \beta}^{\star}$, which can be achieved by changing the needle diameter. For a 
given dimensional $\tilde{\sigma}_{\alpha \beta}$, decreasing the dimensional radius, $\tilde{r}_{c}$ increases $\sigma_{\alpha \beta}^{\star}$. As this promotes a spherical drop shape, a large drop size compared to the capillary diameter is then needed to be able to determine $G$ as well. A large value of $\sigma_{\alpha \beta}^{\star}$ represents the optimal condition for isotropic drop shapes. For elastic drops the influence of the shear modulus $G$ then becomes imperceptible. All in all, the pressure-assisted elastometry performs best and the drop-shape fitting elastometry worst.

For larger values of $\sigma_{\alpha \beta}^{\star}$ stress-fitting elastometry (SFE) starts to show pronounced oscillations. As the neck region decreases, the influence of the shear modulus on the shape is less pronounced. In the opposite limit, for small $\sigma^{\star}$, SFE shows also oscillations for $G$. For small values of $\sigma_{\alpha \beta}^{\star}$ the cosine term in eq.(7) is small and, consequently, small variations in $d \sigma^{s} / d s$ cause oscillatory behavior in $\sigma^{r}$. At this point we are not sure if there is a physical reason for this singular behavior or if this is merely due to an inconvenient mathematical formulation. The alternative formulation (eq.(18) by Danov et al. [26]), which does not share the same singular behavior, leads to equivalent results owing to small variations in the meridional curvature that are amplified by the meridional stress.
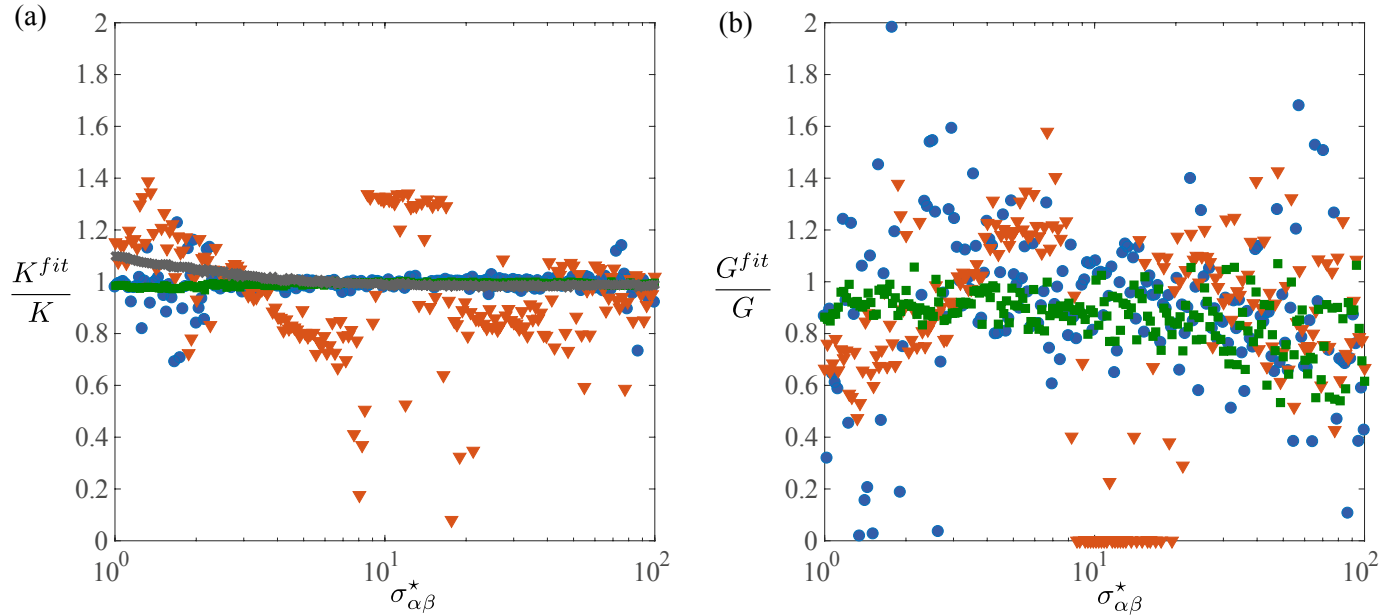

Figure 14: Sensitivity of the different methods to the relative magnitude of $\sigma_{\alpha \beta}^{\star}$. Fitted normalized moduli versus the initial state surface tension $\sigma_{\alpha \beta}^{\star}$. Stress-fitting elastometry (blue), drop shape fitting elastometry (red), pressure-assisted elastometry (green) and the CPT method (grey) for K. Interface parameters are $v^{\star}=4.5 \sigma_{\alpha \beta}^{\star}$ and $5 \%$ area compression $K=2 / 3 \sigma_{\alpha \beta}^{\star}$ and $G=3 / 4 K$.

Pressure-assisted elastometry and stress-fitting elastometry, are intrinsically performing well. However, in the calculations the pressure was known exactly, where under experimental conditions this may not be the case. Figure 15 shows the relative errors when using Stress-fitting elastometry or pressureassisted elastometry against an error or perturbation of the pressure. The errors increase rapidly for small pressure deviations. Stress-fitting elastometry is more robust and an error of about $10 \%$ is introduced for $\Delta p= \pm 1$, however, the pressure-assisted elastometry diverges rapidly and especially the shear modulus $G$ becomes erroneous for small deviations. For practical measurements this means precise elastometry requires accurate determination of the hydrostatic pressure of the liquid in the cuvette, for instance. Furthermore, it requires the use of a pressure transducer whose static noise lies within tight margins. Stress-fitting elastometry is less sensitive against these deviations because differences of stresses are evaluated and changing the pressure shifts at first order the whole stress curve, which does influence the stress difference.

Finally, in the calculations so far, the initial state has been provided exactly, and this initial state was chosen to be ideal, meaning free of extra and deviatoric stresses. The effect of deviations from a stress-free reference state on the determination of $K^{f i t}$ and $G^{f i t}$ are shown in figure 16 . As can be seen in this figure, the drop shape-fitting elastometry, as developed by Knoche et al. but modified with a Hencky strain measure, again is the most prone to errors, leading to unacceptable errors upon small deviations from a stress-free initial state. Pressure-assisted elastometry can handle minor variations of the initial compression as far as $K^{f i t}$ is concerned, but fails to accurately determine the deviatoric stresses and thereby $G^{f i t}$, as the strain in the neck region is most affected by the initial state. Stress-fitting 


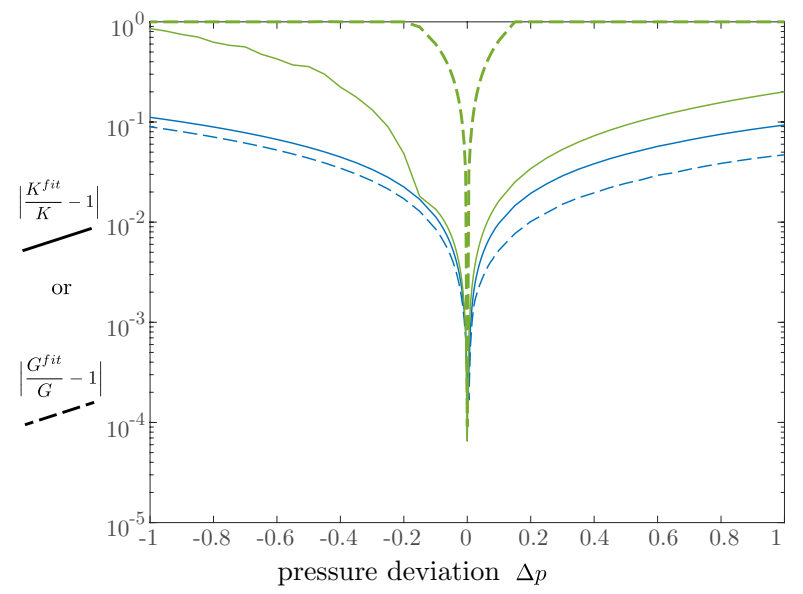

Figure 15: Sensitivity to errors in the pressure measurement on the relative errors on $K$ and $G$, obtained from syntheticnumeric shapes using Stress-fitting elastometry (blue) and pressure-assisted elastometry (green) $\left(\sigma_{\alpha \beta}^{\star}=6, K=4\right.$ and $G=3)$. Full lines show the error for $K$ and dashed lines the error for $G$.

elastometry was designed to work with stressed reference states, as it compares local stress differences. This constitutes a major advantage of stress-fitting elastometry relative to other methods.
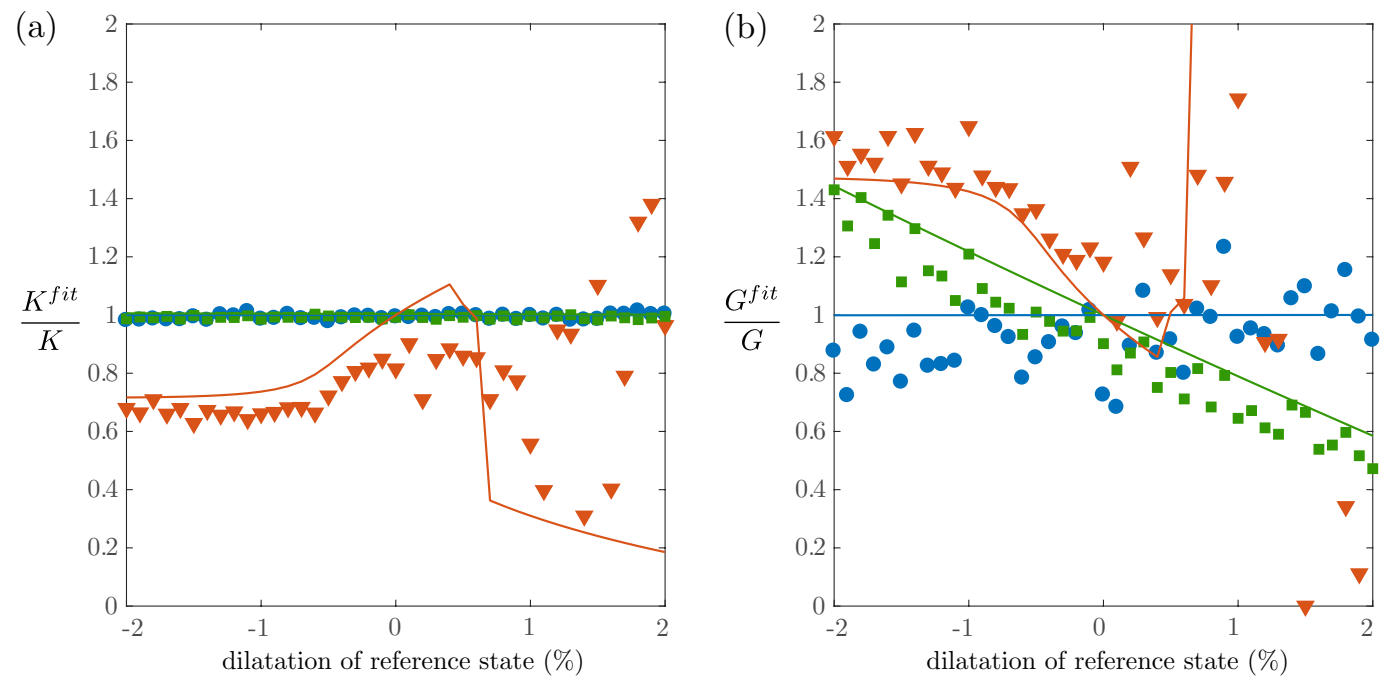

Figure 16: Sensitivity to deviations from an isotropic reference state. The normalized moduli are plotted against the dilation/compression ratio of the initial state. Stress-fitting elastometry (blue), drop shape fitting elastometry (red) and CPT (green). $\left(\sigma_{\alpha \beta}^{\star}=6, K=4\right.$ and $\left.G=3\right)$. Symbols for synthetic-images, lines purely numeric shapes.

In actual experiments the different sources of error that we have analyzed here one by one, will appear in a combined fashion. Very likely this will hinder even further the convergence to the correct solution for the drop-shape fitting elastometry and pressure-assisted elastometry. With fewer unknowns and being, therefore, less plagued by non-linearities, we believe that stress-fitting elastometry should be more robust for actual measurements.

\section{Summary and conclusion}

The simplicity of the experimental configuration makes pendant drop experiments appealing for determining the surface tension and the interfacial rheological parameters. For surface-tension measurements, drop-shape analysis is known to be a robust method, and this was confirmed here by comparing this technique with a method based on a local force balance. When elastic (or fast deformed viscoelastic) interfaces are present, the interfaces may not deform uniformly anymore. The capillary holding the drop 
or bubble typically imposes a constraint, which restricts the surface deformation in radial direction. When the interface has a non-zero surface modulus $G$, this will entail anisotropic stress states. The deformation is then no longer uniform, $\lambda^{s}$ and $\lambda^{r}$ no longer constant, and the stresses $\sigma^{s}$ and $\sigma^{r}$ will differ, in particular in the neck region of the capillary. Due to symmetry, the stress will still be isotropic at the drop apex. But, in addition to the equilibrium surface tension, there will now also be a strain-dependent isotropic contribution to the stress.

If one is only interested in the surface tension and the compression modulus, the experiments can be performed at relatively high values of the non-dimensional surface tension $\sigma_{\alpha \beta}=\frac{\tilde{\sigma}_{\alpha \beta}}{\rho \tilde{g} \tilde{r}_{c}}$. The drop and bubble will then be outside of the capillary, and the curvature will be constant over most part of the drop surface. This implies that normal drop-shape analysis will function poorly $\left(W_{o}<0.4\right)$ and an additional measurement of the pressure is required, as in capillary-pressure tensiometry (CPT). However, a detailed analysis of the deformation profiles and fitting errors shows that the hemispherical geometry, which is typically adopted in such experiments is not ideal. Yet a CPT device will provide accurate data on the compression modulus with only few percent of error when the initial state has a small neck region as is the case for drops with large $\sigma_{\alpha \beta}^{\star}$.

When classical drop-shape analysis is applied using the isotropic Young-Laplace equation, the errors are significant. Drop-shape analysis requires drops that are sufficiently deformed by gravity and will only have the chance to capture the surface tension correctly for very small ratios of $G / K$. Moreover, it is a rather inadequate method to assess whether this requirement is met experimentally. Drop-shape fitting elastometry, which invokes a constitutive model, as introduced by Knoche et al. [25], did easily diverge, leading to a wrong solution because the non-linear shape could not be identified unambiguously, especially at small strains. When the pressure inside the drop is used as additional information, e.g. pressure-assisted elastometry, better results are obtained, but a quasi linear strain measure needs to be used instead of the infinitesimal strain measure used by Knoche et al. [25]. However, both the drop-shape fitting elastometry and pressure-assisted elastometry require the knowledge of an unstrained reference state. To circumvent this difficulty, stress-fitting elastometry was developed, which combines a local force balance method $[11,26]$ with an adequate constitutive relation. Stress-fitting elastometry method showed more scatter of the data and did not converge as well due to the direct stress-fitting approach that may amplify fit errors. However, it was shown that stress-fitting elastometry is more robust against perturbations of pressure signal and does not require the knowledge of an unstrained initial state and may be more useful than the other two algorithms in practical applications. Moreover, the plots of the stress profiles are the best indicators to see if extra and deviatoric stresses matter. With all models we observed that the compression modulus could be obtained with a higher precision than the shear modulus because the information is encoded in the whole interface, whereas the shear modulus acts predominantly in a region near the capillary, where the interface is discontinuous and, therefore, the shape sampling is less precise.

In order to maximize the precision and robustness we presented a novel algorithm for the inverse solution of the surface tension or rheological data from pendant drop measurements. A filtering scheme that allows to extract precise and smooth interface coordinates was introduced first. For the classical drop-shape analysis or capillary-pressure tensiometry this is of negligible influence. However, for the elastometric methods this is beneficial and especially for direct methods that solve for the surface tension and surface stresses such a smoothing step is essential. Apart from large strain measures a further complication is introduced for instance by Neo-Hookean material models, which for instance incorporate a change of the moduli as the interface is compressed. A correct treatment would require the definition of a reference state or a reference surface coverage. Otherwise the modulus will be ambiguous. Although a non-linear model would give more reliable results as it could allow for larger strains we have seen in our results that due to the limited precision pendant drop elastometry may not be a suitable tool to identify an appropriate non-linear model.

Whereas the discussion in this work was limited to step strain experiments on drop and bubbles, the key issues carry over to oscillatory pendant drop experiments, when the interface is viscoelastic. The static case for elastic interfaces represents the limit of a high Deborah number, when the time scale of the deformation is fast compared to the relaxation time of the interface. Of course in that case hydrodynamics and mass transfer kinetics with the substrate may lead to additional complexity. 
In presenting the numerical method not all technical aspects have been presented in full detail. A set of MATLAB routines has been made available online (tensiomet. sourceforge.net) under an open source license.

\section{Acknowledgements}

Mathias Nagel would like to thank Dr. E. Boujo (ETH Zurich) for suggesting the projection on an orthogonal basis and is indebted to Prof. J. Hoepffner (UPMC Paris) for his introduction to the Spectral Method. We also thank Prof. P. Anderson (TU Eindhoven) and Prof. G. Fuller (Stanford University) for fruitful discussions.

\section{Appendix A. Conversion between rheological parameters in $2 \mathrm{D}$}

For conversion between a $2 D$ Youngs modulus $E$ with Poisson ratio $\nu$ and compression and shear moduli $K$ and $G$, the following relations can be used [37]:

$$
K=\frac{E}{2(1-\nu)}, \quad G=\frac{E}{2(1+\nu)}, \quad E=\frac{4 G K}{G+K} \quad \text { and } \quad \nu=\frac{K-G}{K+G} .
$$

\section{Appendix B. Numerical formulation of the isotropic Laplace equation}

As mentioned in section 3.1 the length of the computational domain $s$ is not known a priori and a stretch parameter $C$ relates $s$ to a fixed approximate $s_{0}$. The arc-length $s$ does not enter any of the equations of interest directly but only through the derivative operator $\frac{d f}{d s}$. Using the derivative operator for $s_{0}, \frac{d f}{d s_{0}}$, together with the definition $s=s_{0} / C$, we get:

$$
\frac{d f}{d s}=C \frac{d f}{d s_{0}}=C f^{\prime}
$$

We abbreviate the derivative $\frac{d f}{d s_{0}}$ by $f^{\prime}$ and equations $(2 \mathrm{a}, \mathrm{b})$ and (5) become:

$$
\begin{aligned}
C r^{\prime}=\cos (\psi), & r(0)=0, \\
C z^{\prime}=\sin (\psi), & z\left(s_{0}\right)=0, \\
p-z=\sigma\left(C \psi^{\prime}+\frac{\sin (\psi)}{r}\right), & \psi(0)=0 .
\end{aligned}
$$

In order to solve these equations numerically, they are linearized and discretized. We use Newton iteration, where the non-linear equation system $F(x+\delta x)=0$ is linearized with the Jacobian $L=d F / d x$ to $L \delta(x)=-F(x)$. For example, eq.(A.2):

$$
r^{\prime} \delta C+C \delta r^{\prime}+\sin (\psi) \delta \psi=-\left(C r^{\prime}-\cos (\psi)\right) .
$$

After discretization the differential operator $(I)$ is substituted by a matrix $D_{i j}$ and the differential equation becomes thus an algebraic equation.

$$
(\mathbf{D r}) \delta C+C \mathbf{D} \delta \mathbf{r}+\sin (\boldsymbol{\psi}) \delta \boldsymbol{\psi}=-(C \mathbf{D r}-\cos (\boldsymbol{\psi})) .
$$

760

$2 N+1$ unknowns appearing $\mathbf{r}, \boldsymbol{\psi}$ and $C$ in $N$ equations. For the solution of the isotropic problem the coupling of the equations $2 \mathrm{a}$, b and 5 results in a $3 N+1 \times 3 N+1$ equation system for the forward problem and a $4 N \times 3 N+2$ equation system for the inverse problem. In the inverse problem the arc-length $s$ is given and therefore $C=1$. One can also allow $C$ to be variable with no pronounced effect.

The iterative scheme is closed by updating the variables i.e. $r_{n e w}=r_{\text {old }}+\alpha \delta r$. The relaxation $\alpha=0.5$ and for the fitting elastic interfaces $\alpha=0.1$ for increased robustness. 


\section{Appendix C. Numerical formulation of the generalized Laplace equations for elastic inter- faces}

The elastic Laplace equations have a given arc-length coming from the unstrained reference state and so $C=1$. However the problem formulations for the forward and inverse problem are quite different. We begin with the forward problem, where the derivatives are based on the reference state and therefore are modified for the elastic state. We recall:

$$
\lambda^{s}=\frac{d s}{d s^{\star}} \rightarrow \frac{d f}{d s}=\frac{d s^{\star}}{d s} \frac{d f}{d s^{\star}}=\frac{1}{\lambda^{s}} \frac{d f}{d s^{\star}}=\frac{1}{\lambda^{s}} f^{\prime} .
$$

The resulting equation system for the forward problem comprises $7 N$ unknowns:

$$
\begin{array}{rc}
r^{\prime}=\lambda^{s} \cos (\psi), & r(0)=0, \\
z^{\prime}=\lambda^{s} \sin (\psi), & z\left(s_{0}\right)=0, \\
\lambda^{s}(p-z)=\sigma^{s} \psi^{\prime}+\sigma^{r} \lambda^{s} \frac{\sin (\psi)}{r}, & \psi(0)=0, \\
r \sigma^{s \prime}+\lambda^{s} \cos (\psi)\left(\sigma^{r}-\sigma^{s}\right)=0, & \sigma^{s \prime}(0)=0, \\
\sigma^{s}-\sigma_{\alpha \beta}^{\star}=K \ln \left(\lambda^{s} \lambda^{r}\right)+G \ln \left(\frac{\lambda^{s}}{\lambda^{r}}\right), & \\
\sigma^{r}-\sigma_{\alpha \beta}^{\star}=K \ln \left(\lambda^{s} \lambda^{r}\right)+G \ln \left(\frac{\lambda^{r}}{\lambda^{s}}\right), & \\
r=\lambda^{r} r^{\star}, & \lambda^{r}(0)=\lambda^{s}(0) .
\end{array}
$$

The inverse problem differs from the forward problem because it is more stable when the deformed state is the reference state. This stability is mainly due to the fact that the strain measures $\lambda$ do not appear in the derivatives anymore, which make the equation system less non-linear. Therefore in the following the derivative and integral operator are from the deformed state.

$$
\begin{array}{r}
r^{\prime}=\cos (\psi), \\
z^{\prime}=\sin (\psi), \\
\sigma^{s} \psi^{\prime}+\sigma^{r} \frac{\sin (\psi)}{r}=p-z, \\
r \sigma^{s \prime}+\cos (\psi)\left(\sigma^{r}-\sigma^{s}\right)=0, \\
\sigma^{s}-\sigma_{\alpha \beta}^{\star}=K \ln \left(\lambda^{s} \lambda^{r}\right)+G \ln \left(\frac{\lambda^{s}}{\lambda^{r}}\right), \\
\sigma^{r}-\sigma_{\alpha \beta}^{\star}=K \ln \left(\lambda^{s} \lambda^{r}\right)+G \ln \left(\frac{\lambda^{r}}{\lambda^{s}}\right), \\
r=\lambda_{r} r^{\star} .
\end{array}
$$

The last equation contains a subtlety that needs careful attention otherwise the method will not be convergent. The meridional deformation $\lambda^{s}$ follows from the solution process whereas $\lambda^{r}$ is accessible from the imaging process. However the location where $r^{\star}$ is compared to $r$ (when evaluating $\lambda^{r}$ ) depends on $\lambda^{s}$. The fixed grid on the deformed reference state is related to locations on the unstrained shape by $s^{\star}=\int_{0}^{s} 1 / \lambda^{s}(t) d t$. We hence linearize the equation (C.15) for $\lambda^{r}$ as:

$$
r(s)=\lambda^{s} r^{\star}\left(s^{\star}\right)=\lambda^{s}\left(r^{\star}+\frac{d r^{\star}}{d s^{\star}} \delta s^{\star}\right)=\lambda^{s}\left(r^{\star}-\frac{d r^{\star}}{d s^{\star}} \int_{0}^{s} \frac{\delta \lambda^{s}}{\lambda^{s} 2} d t\right),
$$

To avoid confusion we clarify that in the last term $\lambda^{s^{2}}$ is $\lambda^{s}$ squared. We introduce the inverse of the differentiation matrix $\mathbf{W}=\mathbf{D}^{-1}$ as the discrete integral operator. Finally equation (C.15) is obtained in its discretized form:

$$
\delta \mathbf{r}-\mathbf{r}^{\star} \delta \lambda^{r}+\lambda^{r} \frac{d r^{\star}}{d s^{\star}} \mathbf{W} \frac{\delta \lambda_{s}}{\lambda^{s} 2}=\lambda^{r} \mathbf{r}^{\star}-\mathbf{r} .
$$

We evaluate $\frac{d r^{\star}}{d s^{\star}}$ from the acquired data on a fixed grid on the unstrained state. As $\lambda^{s}$ is evolved $\frac{d r^{\star}}{d s^{\star}}$ and $\mathbf{s}^{\star}$ is interpolated on updated positions $\mathbf{s}^{\star}=\mathbf{W} \mathbf{s}$. 


\section{Appendix D. Volume and Area from discrete data}

We compute the volume and surface area from the numeric data with the following equations.

$$
\begin{array}{r}
v=\int_{0}^{s} \pi r^{2} \frac{d z}{d s} d s=\int_{0}^{s} \pi r^{2} \sin (\psi) d s=\pi \mathbf{w} r^{2} \sin (\psi), \\
a=\int_{0}^{s} 2 \pi r d s=2 \pi \mathbf{w} r .
\end{array}
$$

Here $\mathbf{w}$ is the last line of the integral operator $\mathbf{W}$, which corresponds to integration over the entire domain. By considering the pressure $p$ as a free variable and adding one of the above equations in discretized from to the equation system in Appendix B and Appendix C, we can also compute drop shapes with imposed volume or surface area.

\section{Appendix E. Block matrix diagonalization}

The correct way to pose the inverse problem is to ask the solution to fulfill the Laplace law exactly and to accommodate the residuum only in the fitting error. In this manner the obtained shape is the closest Young-Laplace shape that fits the imaged shape. One way to achieve this is to use the Schur complement. This amounts to inverting the matrix related to the Laplace equation, geometric identities and if applicable to the material models, which is hence solved exactly, and to build a rectangular matrix that contains $N$ equations from the collocation information and the unknowns $\sigma_{\alpha \beta}, p$ in order to minimize the fitting error.

For the isotropic interface this has no impact, but for the elastic interface, due to the coupling of $\sigma$ and $\lambda$, the solution can become oscillatory and diverge when the fit error perturbs the differential equations. We have, therefore, applied the block diagonalization to the inverse problem of the elastic interfaces and condensed the matrix to $N$ equations for the collocation and 3 unknowns $K, G$ and if applicable $p$.

If one considers the overdetermined equation system as an assembly of submatrices, where $A$ is the determined (generalized) Laplace equations, $B$ the influence of the extra parameters like $p, K$ and $G$ on the Laplace equations, $C$ the collocation matrix and $Z$ being an empty matrix. Then there is a right hand side $a$ from the (generalized) Laplace equations and $c$ for the collocation equations and a solution vector $u$ for the interface position, stress and strain and the extra parameters $v$.

$$
\left(\begin{array}{ll}
\mathbf{A} & \mathbf{B} \\
\mathbf{C} & \mathbf{Z}
\end{array}\right)\left(\begin{array}{l}
\mathbf{u} \\
\mathbf{v}
\end{array}\right)=\left(\begin{array}{l}
\mathbf{a} \\
\mathbf{c}
\end{array}\right)
$$

One can use the inverse of the determined problem $\mathbf{A}^{-1}$ to block diagonalize the matrix and to obtain the overdetermined matrix:

$$
\left(\mathbf{C A}^{-1} \mathbf{B}\right) \mathbf{v}=\mathbf{C A}^{-1} \mathbf{a}-\mathbf{b}, \quad \mathbf{u}=\mathbf{A}^{-1}(\mathbf{a}-\mathbf{B v}) .
$$

795 One first solves for the extra parameters $\mathbf{v}$ from the overdetermined matrix. Hereafter the interfacial problem for $\mathbf{u}$ is imposed exactly.

\section{Appendix F. Convergence}

For the shape fitting methods, figure F.17 shows the convergence of the relative error for $K$ and $G$ when increasing the number of unknowns $N$ or number of discrete points on the drop interface. The interface has been computed with 100 points sampled equidistantly and is then interpolated on a coarser grid. One observes that pressure-assisted elastometry converges fastest and that stress-fitting elastometry shows the highest residual error. All methods converge at about 30 point with the exponential convergence due to the accuracy of the spectral method.

The sampling of the shape with Chebyshev filtering is demonstrated in Figure F.18, where the synthetic shape is translated into a grey value image. The resolution is adapted such that the maximal drop radius is covered by 1000 pixels. As explained in section 2.1 the highest order of Chebyshev modes that is retained is the one that yields more than $10 \%$ decrease of the fit error. For the sampled syntheticimages the relative errors are generally much higher than for synthetic-numeric profiles. Stress-fitting 


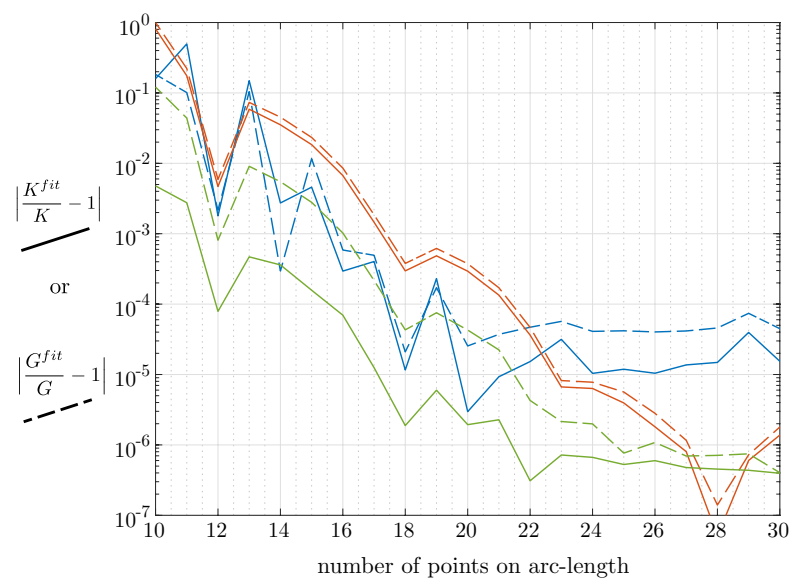

Figure F.17: Relative errors on $K$ and $G$ from synthetic-numeric shapes as a function of the number of sampling points on the arc. Direct method: Stress-fitting elastometry (blue), Shape fitting: Drop shape fitting elastometry (red) and pressureassisted elastometry (green). Full lines show the error for $K$ and dashed lines the error for $G$. Parameters $\sigma_{\alpha \beta}^{\star}=6, K=4$ and $G=3$.

elastometry is shown to be less robust because of the direct evaluation of the surface stresses that require smoothness and accuracy. Nevertheless the optimal Chebyshev order is a useful indicator for the quality of the interface.

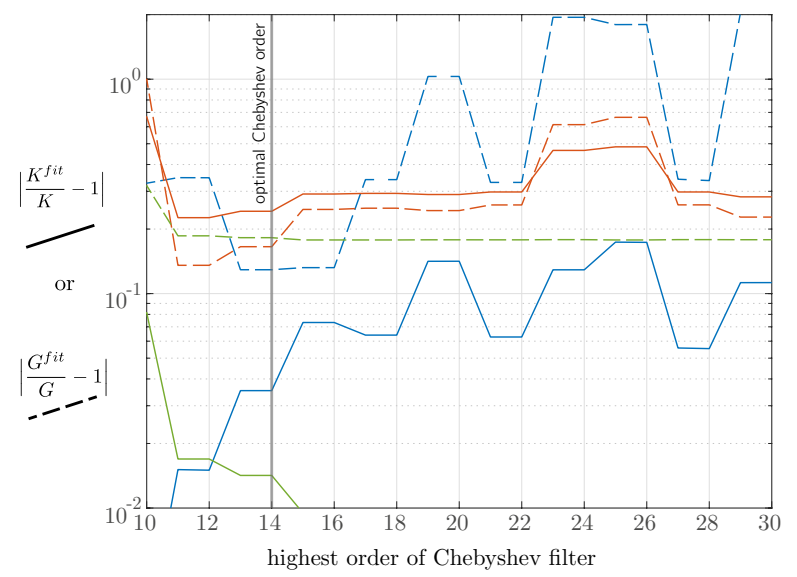

Figure F.18: Example for fitting $K$ and $G$ from synthetic-image shapes. Parameters $\sigma_{\alpha \beta}^{\star}=6, K=4$ and $G=3$. Shown are the relative errors against the number of used Chebyshev modes to filter the interface for three methods: Stress-fitting elastometry (blue), drop shape fitting elastometry (red) and pressure-assisted elastometry (green). Full lines show the error for $K$ and dashed lines the error for $G$. The highest order that contributes to a decreased shape sampling error is 14 .

\section{Appendix G. Hencky strain versus infinitesimal strain measure}

To demonstrate that the Hencky strain is more suitable for the type of finite strain deformations which are resolvable in pendant drop experiments as compared to the infinitesimal strain measure used by Knoche et al. [25], Figure G.19 compares the relative error obtained when using a linear Hookean model to fit shapes that are calculated versus the quasi linear Hencky strain based model. We use the proper inverse formulations for elastometry based on drop shape fitting and pressure assisted. Similar to the method by Knoche et al. except that our implementation is a based on an inverse problem, whose convergence we proved in the previous figure. Acknowledging that the deviation is dependent on the material parameters $K$ and $G$ we can see, for this case, that both methods deviate to about 5 to $15 \%$ for $20 \%$ compression. 


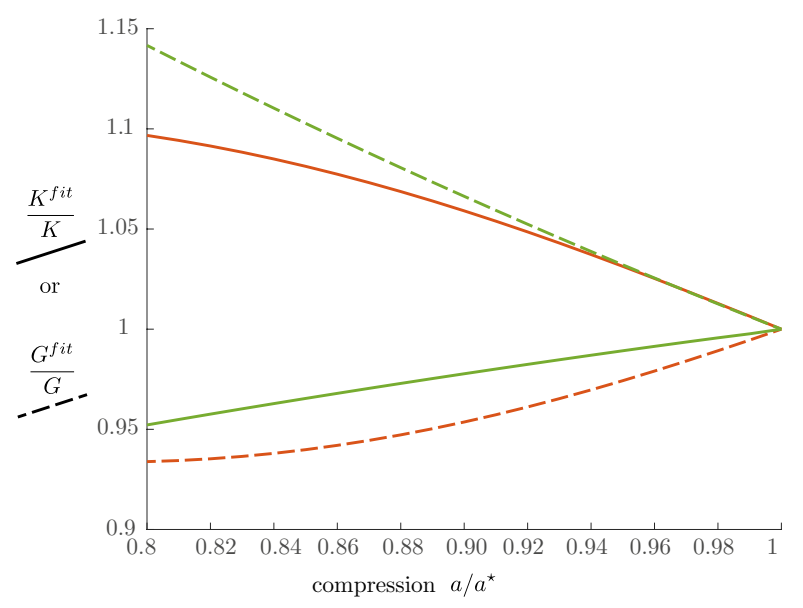

Figure G.19: Example for fitting $K$ and $G$ with a linear Hookean model to synthetic-numeric shapes using-linear Hookean model. Parameters $\sigma_{\alpha \beta}^{\star}=6, K=4$ and $G=3$. Shown are the normalized moduli against the compression ratio. Drop shape fitting elastometry is shown red and pressure-assisted elastometry green. Full lines show the error for $K$ and dashed lines the error for $G$.

\section{References}

[1] A. M. Worthington, On pendent drops, Proceedings of the Royal Society of London 32 (212-215) (1881) 362-377.

[2] Y. Rotenberg, L. Boruvka, A. Neumann, Determination of surface tension and contact angle from the shapes of axisymmetric fluid interfaces, Journal of Colloid and Interface Science 93 (1) (1983) 169 - 183.

[3] J. Andreas, E. Hauser, W. Tucker, Boundary tension by pendant drops, The Journal of Physical Chemistry 42 (8) (1938) 1001-1019.

[4] S. Fordham, On the calculation of surface tension from measurements of pendant drops, Proceedings of the Royal Society of London A: Mathematical, Physical and Engineering Sciences 194 (1036) (1948) 1-16. doi:10.1098/rspa.1948.0063.

[5] O. Río, A. Neumann, Axisymmetric drop shape analysis: Computational methods for the measurement of interfacial properties from the shape and dimensions of pendant and sessile drops, Journal of Colloid and Interface Science 196 (2) (1997) $136-147$.

[6] M. Hoorfar, A. W. Neumann, Recent progress in Axisymmetric Drop Shape Analysis (ADSA), Advances in Colloid and Interface Science 121 (1-3) (2006) 25-49.

[7] J. D. Berry, M. J. Neeson, R. R. Dagastine, D. Y. Chan, R. F. Tabor, Measurement of surface and interfacial tension using pendant drop tensiometry, Journal of Colloid and Interface Science 454 (2015) $226-237$.

[8] S. M. Saad, Z. Policova, A. W. Neumann, Design and accuracy of pendant drop methods for surface tension measurement, Colloids and Surfaces A: Physicochemical and Engineering Aspects 384 (1-3) (2011) $442-452$.

[9] S. Sugden, Xcvii.-the determination of surface tension from the maximum pressure in bubbles, J. Chem. Soc., Trans. 121 (1922) 858-866.

[10] G. Enhorning, Pulsating bubble technique for evaluating pulmonary surfactant, Journal of Applied Physiology 43 (2) (1977) 198-203.

[11] F. Peters, D. Arabali, Interfacial tension between oil and water measured with a modified contour method, Colloids and Surfaces A: Physicochemical and Engineering Aspects 426 (2013) $1-5$.

[12] A. Neumann, J. Spelt, Applied Surface Thermodynamics, Marcel Dekker, New York, 1996.

[13] J. Holgado-Terriza, J. Gómez-Lopera, P. Luque-Escamilla, C. Atae-Allah, M. Cabrerizo-Vílchez, Measurement of ultralow interfacial tension with ADSA using an entropic edge-detector, Colloids and Surfaces A: Physicochemical and Engineering Aspects 156 (1-3) (1999) 579 - 586.

[14] Y. Rotenberg, L. Boruvka, A. Neumann, Determination of surface tension and contact angle from the shapes of axisymmetric fluid interfaces, Journal of Colloid and Interface Science 93 (1) (1983) 169 - 183.

[15] R. Miller, R. Sedev, K.-H. Schano, C. Ng, A. Neumann, Relaxation of adsorption layers at solution/air interfaces using axisymmetric drop-shape analysis, Colloids and Surfaces 69 (4) (1993) $209-216$.

[16] D. Kwok, D. Vollhardt, R. Miller, D. Li, A. Neumann, Axisymmetric drop shape analysis as a film balance, Colloids and Surfaces A: Physicochemical and Engineering Aspects 88 (1) (1994) $51-58$.

[17] J. Li, R. Miller, R. Wostneck, H. Mowald, A. Neumann, Use of pendent drop technique as a film balance at liquid/liquid interfaces, Colloids and Surfaces A: Physicochemical and Engineering Aspects 96 (3) (1995) 295 - 299.

[18] N. A. Alexandrov, K. G. Marinova, T. D. Gurkov, K. D. Danov, P. A. Kralchevsky, S. D. Stoyanov, T. B. Blijdenstein, L. N. Arnaudov, E. G. Pelan, A. Lips, Interfacial layers from the protein HFBII hydrophobin: Dynamic surface tension, dilatational elasticity and relaxation times, Journal of Colloid and Interface Science 376 (1) (2012) 296 - 306.

[19] F. Boulogne, F. Giorgiutti-Dauphine, L. Pauchard, The buckling and invagination process during consolidation of colloidal droplets, Soft Matter 9 (2013) 750-757.

[20] C. Gu, L. Botto, Direct calculation of anisotropic surface stresses during deformation of a particle-covered drop, Soft Matter 12 (2016) 705-716.

[21] N. J. Alvarez, L. M. Walker, S. L. Anna, Diffusion-limited adsorption to a spherical geometry: The impact of curvature and competitive time scales, Phys. Rev. E 82 (2010) 011604. doi:10.1103/PhysRevE.82.011604.

[22] C. Balemans, M. A. Hulsen, P. D. Anderson, Modeling of complex interfaces for pendant drop experiments, Rheologica Acta 55 (10) (2016) 801-822. 
[23] D. Carvajal, E. J. Laprade, K. J. Henderson, K. R. Shull, Mechanics of pendant drops and axisymmetric membranes, Soft Matter 7 (22) (2011) 10508-12.

[24] J. K. Ferri, P. A. L. Fernandes, J. T. McRuiz, F. Gambinossi, Elastic nanomembrane metrology at fluid-fluid interfaces using axisymmetric drop shape analysis with anisotropic surface tensions: deviations from Young-Laplace equation, Soft Matter 8 (2012) 10352-10359.

[25] S. Knoche, D. Vella, E. Aumaitre, P. Degen, H. Rehage, P. Cicuta, J. Kierfeld, Elastometry of Deflated Capsules: Elastic Moduli from Shape and Wrinkle Analysis, Langmuir 29 (40) (2013) 12463-12471.

[26] K. D. Danov, R. D. Stanimirova, P. A. Kralchevsky, K. G. Marinova, N. A. Alexandrov, S. D. Stoyanov, T. B. J. Blijdenstein, E. G. Pelan, Capillary meniscus dynamometry - Method for determining the surface tension of drops and bubbles with isotropic and anisotropic surface stress distributions, Journal of Colloid and Interface Science 440 (C) (2015) $168-178$.

[27] J. T. Petkov, T. D. Gurkov, B. E. Campbell, R. P. Borwankar, Dilatational and shear elasticity of gel-like protein layers on air/water interface, Langmuir 16 (8) (2000) 3703-3711.

[28] L. N. Trefethen, Spectral Methods in MATLAB, SIAM, 2000.

[29] L. M. C. Sagis, Dynamic properties of interfaces in soft matter: Experiments and theory, Rev. Mod. Phys. 83 (2011) 1367-1403.

[30] G. G. Fuller, J. Vermant, Complex fluid-fluid interfaces: Rheology and structure, Annual Review of Chemical and Biomolecular Engineering 3 (1) (2012) 519-543. arXiv:http://dx.doi.org/10.1146/annurev-chembioeng-061010-114202.

[31] L. Anand, On H. Hencky's approximate strain-energy function for moderate deformations, Journal of Applied Mechanics 46 (1) (1979) 78-82. doi:10.1115/1.3424532.

[32] T. Verwijlen, L. Imperiali, J. Vermant, Separating viscoelastic and compressibility contributions in pressure-area isotherm measurements, Advances in Colloid and Interface Science 206 (2014) 428 - 436, manuel G. Velarde.

[33] R. Gunde, A. Kumar, S. Lehnert-Batar, R. Mäder, E. J. Windhab, Measurement of the surface and interfacial tension from maximum volume of a pendant drop, Journal of Colloid and Interface Science 244 (1) (2001) 113 - 122.

[34] A. P. Kotula, S. L. Anna, Regular perturbation analysis of small amplitude oscillatory dilatation of an interface in a capillary pressure tensiometer, Journal of Rheology 59 (1) (2015) 85-117. doi:10.1122/1.4902546.

[35] S. M. Saad, A. W. Neumann, Total Gaussian curvature, drop shapes and the range of applicability of drop shape techniques, Advances in Colloid and Interface Science 204 (2014) 1 - 14.

[36] J. Hegemann, S. Knoche, S. Egger, M. Kott, S. Demand, A. Unverfehrt, H. Rehage, J. Kierfeld, Pendant capsule elastometry, submitted to J. Colloid Interface Sci. URL http://arxiv.org/abs/1705.11134

[37] I. Jasiuk, J. Chen, M. F. Thorpe, Elastic Moduli of Two Dimensional Materials With Polygonal and Elliptical Holes, Applied Mechanics Reviews 47 (1S) (1994) S18-S28. 